Pacific

Journal of

Mathematics

DEFORMATIONS OF THE GYROID AND LIDINOID MINIMAL SURFACES

ADAM G. WEYHAUPT

Volume $235 \quad$ No. 1

March 2008 


\title{
DEFORMATIONS OF THE GYROID AND LIDINOID MINIMAL SURFACES
}

\author{
ADAM G. WEYHAUPT
}

\begin{abstract}
The gyroid and Lidinoid are triply periodic minimal surfaces of genus three embedded in $\mathbf{R}^{\mathbf{3}}$ that contain no straight lines or planar symmetry curves. They are the unique embedded members of the associate families of the Schwarz $P$ and $H$ surfaces. We prove the existence of two 1-parameter families of embedded triply periodic minimal surfaces of genus three that contain the gyroid and a single 1-parameter family that contains the Lidinoid. We accomplish this by using the flat structures induced by the holomorphic 1-forms $G d h,(1 / G) d h$, and $d h$. An explicit parametrization of the gyroid using theta functions enables us to find a curve of solutions in a two-dimensional moduli space of flat structures by means of an intermediate value argument.
\end{abstract}

\section{Introduction}

The gyroid was discovered by Alan Schoen [1970], a NASA crystallographer interested in strong but light materials. Among its most curious properties was that, unlike other known surfaces at the time, the gyroid contains no straight lines or planar symmetry curves [Karcher 1989; Große-Brauckmann and Wohlgemuth 1996]. Soon after, Bill Meeks [1975] discovered a 5-parameter family of embedded genus three triply periodic minimal surfaces.

Theorem 1.1 [Meeks 1975]. There is a real five-dimensional family $V$ of periodic hyperelliptic Riemann surfaces of genus three. These are the surfaces that can be represented as two-sheeted covers of $S^{2}$ branched over four pairs of antipodal points. There exist two distinct isometric minimal embeddings for each $M_{3} \in V$.

Meeks's family contains many known examples of genus three triply periodic minimal surface, including the classical $P, D$, and $C L P$ surfaces. Most members were previously undiscovered surfaces, and many have no straight lines and no planar symmetries. Sven Lidin [1990] discovered a related surface, christened by

MSC2000: primary 53A10; secondary 49Q05, 30F30.

Keywords: minimal surfaces, main/gyroid, lidinoid, triply periodic, flat structures.

I am very grateful to my thesis adviser Matthias Weber for his patient help and encouragement. 
Lidin the HG surface but now commonly called the "Lidinoid". Later, GroßeBrauckmann and Wohlgemuth [1996] proved that the gyroid and Lidinoid are embedded.

Every currently known triply periodic minimal surface of genus three except for the gyroid and Lidinoid is deformable, that is, for each triply periodic minimal surface $M$ there is a continuous family of embedded triply periodic minimal surfaces $M_{\eta}$ for $\eta \in(-\epsilon, \epsilon)$ such that $M=M_{0}$, as long as $M$ is neither the gyroid nor the Lidinoid. In general, the lattices may vary with $\eta$, so that generically $\Lambda_{\eta_{1}} \neq \Lambda_{\eta_{2}}$. We are primarily concerned with this question: do there exist continuous deformations of the gyroid and the Lidinoid? In general, the moduli space of genus three triply periodic minimal surface is not understood, and the existence of these deformations would provide more information about the moduli space.

In a series of papers, the crystallographers and physical chemists Fogden, Haeberlein, Hyde, Lidin, and Larsson graphically argue for the existence of two 1parameter families of embedded triply periodic minimal surfaces that contain the gyroid and two additional families that contain the Lidinoid [Fogden et al. 1993; Fogden and Hyde 1999; Lidin and Larsson 1990]. While accompanied by very convincing computer-generated images, their work does not provide an existence proof, and the mathematical landscape is fraught with examples where pictures mislead; see, for example, [Weber 1998].

The goal of this paper is to establish this main result:

Theorem 1.2. For $\eta \in \mathbb{R}^{+}$, there is a one parameter family of minimal embeddings $M_{\eta} \subset \mathbb{R}^{3} / \Lambda_{\eta}$ such that $M_{\eta}$ is an embedded minimal surface of genus three. This family contains the gyroid. Each $M_{\eta}$ admits a rotational symmetry of order 2 .

We will call this family of surfaces the " $t G$ " family following the notation of [Fogden et al. 1993]; the " $t$ " stands for "tetragonal". This theorem shows that the gyroid is deformable. Our other two main theorems prove the existence of a Lidinoid family and an additional gyroid family.

Theorem 1.3. For $\eta \in \mathbb{R}^{+}$, there is a one parameter family of minimal embeddings $r L_{\eta} \subset \mathbb{R}^{3} / \Lambda_{\eta}$ such that $r L_{\eta}$ is an embedded minimal surface of genus three. This family contains the Lidinoid. Each $r L_{\eta}$ admits a rotational symmetry of order 3 .

Theorem 1.4. For $\eta \in \mathbb{R}^{+}$, there is a one parameter family of minimal embeddings $r G_{\eta} \subset \mathbb{R}^{3} / \Lambda_{\eta}$ such that $r G_{\eta}$ is an embedded minimal surface of genus three. This family contains the gyroid. Each $r G_{\eta}$ admits a rotational symmetry of order 3 .

As a consequence of these results, we have shown this:

All currently known examples of genus three triply periodic minimal surfaces admit deformations.

None of these new examples are members of Meeks's family. 


\section{Preliminaries}

2.1. Parametrizing minimal surfaces. For this section, refer to [Dierkes et al. 1992; Osserman 1969; Nitsche 1975] for further details and history. Let $\Omega \subset \mathbb{C}$ denote a simply connected open domain, and let $h=\left(h_{1}, h_{2}, h_{3}\right): \Omega \rightarrow \mathbb{C}^{3}$ be a nonconstant holomorphic map such that $h_{1}^{2}+h_{2}^{2}+h_{3}^{2} \equiv 0$ and $\left|h_{1}(z)\right|^{2}+\left|h_{2}(z)\right|^{2}+$ $\left|h_{3}(z)\right|^{2} \neq 0$ for all $z \in \Omega$. A direct computation shows that

$$
F: \Omega \rightarrow \mathbb{R}^{3}, \quad p \mapsto \operatorname{Re} \int^{p}\left(h_{1} d z, h_{2} d z, h_{3} d z\right)
$$

is a minimal surface $M \subset \mathbb{R}^{3}$. The normal map $N: M \rightarrow S^{2}$ assigns to each point $p \in M$ the normal at $p$. The Gauss map, $G: M \rightarrow \mathbb{C} \cup \infty$ is the stereographic projection of the normal map.

To relate (2-1) to the geometry of the surface, we can rewrite the map as

$$
p \mapsto \operatorname{Re} \int^{p} \frac{1}{2}\left(\frac{1}{G}-G, \frac{i}{G}+i G, 1\right) d h .
$$

The meromorphic function $G$ in (2-2) is the Gauss map

$$
G=-\frac{h_{1}+i h_{2}}{h_{3}} \text {. }
$$

Here $d h$ is a holomorphic differential, often called the height differential. Given any minimal surface $M$, there exists a height differential $d h$ that, along with the Gauss map, provides the above parametrization of a surface patch. Therefore, simply connected surface patches are fully parametrized.

The next result gives us a way to parametrize nonsimply connected surfaces.

Theorem 2.1 [Osserman 1969]. A complete regular minimal surface $M$ having finite total curvature, that is, satisfying $\int_{M}|K| d A<\infty$, is conformally equivalent to a compact Riemann surface $X$ that has finitely many punctures.

Since our triply periodic minimal surfaces $M$ are compact in the quotient $M / \Lambda$, the fundamental domain necessarily has finite total curvature and therefore can be parameterized on a Riemann surface. Instead of using a simply connected domain $\Omega$ and meromorphic functions $h_{1}, h_{2}$, and $h_{3}$, we instead consider three holomorphic 1-forms $\omega_{1}, \omega_{2}$, and $\omega_{3}$ defined on a Riemann surface $X$, again with $\sum \omega_{i}^{2} \equiv 0$ and $\sum\left|\omega_{i}\right|^{2} \neq 0$ (making sense of this first quantity pointwise and locally). We can then define

$$
F: X \rightarrow \mathbb{R}^{3}, \quad p \mapsto \operatorname{Re} \int^{p}\left(\omega_{1}, \omega_{2}, \omega_{3}\right)
$$

with

$$
\omega_{1}=\frac{1}{2}\left(\frac{1}{G}-G\right) d h, \quad \omega_{2}=\frac{i}{2}\left(\frac{1}{G}+G\right) d h, \quad w_{3}=d h .
$$


Since the domain is no longer simply connected, integration of the Weierstrass data over a homotopically nontrivial loop $\gamma$ on $X$ is generically no longer zero. This integration leads to a translational symmetry of the surface. The surface need not, at this point, be embedded or even immersed, so the term "symmetry" is perhaps misleading here. More precisely, if $F(p)=\left(q_{1}, q_{2}, q_{3}\right) \in \mathbb{R}^{3}$ for some choice of path of integration from the base point to $p$, then for any other choice of path of integration, $F(p)=\left(q_{1}, q_{2}, q_{3}\right)+\int_{\gamma}\left(\omega_{1}, \omega_{2}, \omega_{3}\right)$ for some $\gamma \in H_{1}(X, \mathbb{Z})$. We define the period of $\gamma$ by

$$
P(\gamma):=\operatorname{Re} \int_{\gamma}\left(\omega_{1}, \omega_{2}, \omega_{3}\right)
$$

Fr a surface to be immersed and nonperiodic, we must have $P(\gamma)=0$ for all $\gamma \in H_{1}(X, \mathbb{Z})$. For a surface to be triply periodic with lattice $\Lambda \subset \mathbb{R}^{3}$, we must have

$$
P(\gamma) \in \Lambda \text { for all } \quad \gamma \in H_{1}(X, \mathbb{Z})
$$

Note

$$
F_{1}(z)+i F_{2}(z)=-\int_{.}^{z} G d h+\overline{\int^{z} \frac{1}{G} d h}
$$

so the periods can be written as

$$
P(\gamma)=\left(\begin{array}{c}
\operatorname{Re}\left(-\int_{\gamma} G d h+\overline{\int_{\gamma} \frac{1}{G} d h}\right) \\
\operatorname{Im}\left(-\int_{\gamma} G d h+\overline{\int_{\gamma} \frac{1}{G} d h}\right) \\
\operatorname{Re} \int_{\gamma} d h
\end{array}\right) .
$$

The next lemma gives us a convenient way to generate surfaces.

Lemma 2.2. Let $X$ be a Riemann surface of genus $g$. Let $G: X \rightarrow \mathbb{C} \cup \infty$ be meromorphic, and let $d h$ be a holomorphic 1-form defined on X. Also assume the following:

(1) If $G$ has a zero or pole of order $k$ at $p$, then dh also has a zero at $p$ of order $k$. Conversely, if $d h$ has a zero of order $k$ at $p$, then $G$ must have a zero or pole of order $k$ at $p$.

(2) There exists a lattice $\Lambda \subset \mathbb{R}^{3}$ such that $P(\gamma) \in \Lambda$ for all $\gamma \in H_{1}(X, \mathbb{Z})$.

Then the Weierstrass data $(X, G, d h)$ define an immersed triply periodic minimal surface of genus $g$.

The Weierstrass representation immediately allows for the following well-known construction of a minimal surface. Let $M_{0}$ be a minimal surface defined by Weierstrass data $(X, G, d h)$. We construct a new minimal surface $M_{\theta}$ using Weierstrass data $\left(X, G, e^{i \theta} d h\right)$. Note that the data still satisfies the requirements of Lemma 2.2 , in particular, that $\sum_{j} e^{2 i \theta} \omega_{j}^{2} \equiv 0$. The family of surfaces $M_{\theta}$ for $0 \leq \theta \leq \pi / 2$ 
is called the associate family of $M_{0}$. (Sometimes we say these surfaces $M_{\theta}$ are the Bonnet transform of $M_{0}$; see [Nitsche 1975; Bonnet 1853].) If the period problem is solved for $M_{0}$, it will in general not be solved for $M_{\theta}$, since $P_{M_{\theta}}(\gamma)$ is a linear combination of $P_{M_{0}}(\gamma)$ and $P_{M_{\pi / 2}}(\gamma)$; a generic such linear combination need not be either zero or in a lattice. The associate family plays an crucial role in the construction of the gyroid and Lidinoid.

2.2. Cone metrics. We call a flat structure with cone singularities a cone metric. When it is apparent from context that we are dealing with cone metrics, we will often simply refer to a flat structure. Cone metrics are abundant for Riemann surfaces - every holomorphic 1-form gives rise to a cone metric structure (recall that a Riemann surface of genus $g$ has $g$ linearly independent holomorphic 1-forms).

Proposition 2.3. Let $X$ be a Riemann surface with meromorphic 1-form $\omega$. Let $U_{\alpha}$ be an open covering of $X$ by simply connected sets, with distinguished points $p_{\alpha} \in U_{\alpha}$. Define $g_{\alpha}: U_{\alpha} \rightarrow \mathbb{C}$ by $g_{\alpha}(z)=\int_{p_{\alpha}}^{z} \omega$. Then $\left(U_{\alpha}, g_{\alpha}\right)$ endows $X$ with a cone metric (in fact, a translation structure). If $\omega$ has a zero or pole of order $k$, then this is a cone point of angle $2 \pi(k+1)$.

Proof. First, since $U_{\alpha}$ is simply connected, the integral $\int_{p_{\alpha}}^{z} \omega$ does not depend on the choice of $p_{\alpha}$ - changing $p_{\alpha}$ simply adds a constant.

Away from $\omega$ 's zeros, $g_{\alpha}$ is invertible, and so we have

$$
g_{\alpha \beta}(z)=z+\int_{p_{\beta}}^{p_{\alpha}} \omega=z+\text { const },
$$

which gives $X$ a translation structure.

The developing map of the flat structure is given by $\operatorname{dev}(\gamma)=\int_{\gamma} \omega$. If $\omega$ has a zero or pole at a point $p$ (without loss of generality, $p=0$ ), this developing map extends meromorphically with pre-Schwarzian derivative

$$
\frac{\operatorname{dev}^{\prime \prime}}{\operatorname{dev}^{\prime}}(z)=\frac{d \omega}{\omega} .
$$

In the neighborhood of a zero or pole, we can locally write $\omega=z^{k} h$ for a meromorphic function $h$ with $h(0) \neq 0, \infty$. The residue of the pre-Schwarzian becomes

$$
\operatorname{res}_{0} \frac{\operatorname{dev}^{\prime \prime}}{\operatorname{dev}^{\prime}}(z)=\operatorname{res}_{0} \frac{k}{z}
$$

giving a cone of angle $2 \pi(k+1)$.

2.3. Conformal quotients of triply periodic minimal surfaces. Our principal tool in the study of these surfaces will be taking the quotient of a triply periodic minimal 
surface by a rotational symmetry. We first recall Abel's theorem, the RiemannHurwitz formula, and some corollaries. [Farkas and Kra 1992] contains a full treatment of the Riemann-Hurwitz formula.

Theorem 2.4 (Abel's theorem). Let $\Gamma$ be a lattice in $\mathbb{C}$. There is an elliptic function $f$ on the torus $\mathbb{C} / \Gamma$ with divisor $\sum_{j} n_{j} P_{j}$ if and only if

(1) $\sum_{j} n_{j}=0$, and

(2) $\sum_{j} n_{j} P_{j} \in \Gamma$.

Theorem 2.5 (Riemann-Hurwitz formula). Let $f: N^{\prime} \rightarrow N$ be a (nonconstant) holomorphic map between a compact Riemann surface $N^{\prime}$ of genus $g$ and a compact Riemann surface $N$ of genus $\gamma$. Let the degree of $f$ be $n$. Define the total branching number of the mapping to be $B=\sum_{P \in N^{\prime}} b_{f}(P)$. Then

$$
g=n(\gamma-1)+1+B / 2 .
$$

Corollary 2.6 [Farkas and Kra 1992, V.1.5]. For $1 \neq T \in \operatorname{Aut}(M)$,

$$
|\operatorname{fix}(T)| \leq 2+\frac{2 g}{\operatorname{order}(T)-1}+\frac{2 \gamma \operatorname{order}(T)}{\operatorname{order}(T)-1}
$$

with equality if $\operatorname{order}(T)$ is prime.

Corollary 2.7 [Farkas and Kra 1992, proof of V.1.5].

$$
(2 g-2)=\operatorname{order}(T)(2 \gamma-2)+\sum_{j=1}^{\operatorname{order}(T)-1}\left|\operatorname{fix}\left(T^{j}\right)\right| .
$$

Using these tools, we can describe the quotient of a minimal surface by a rotational symmetry.

Proposition 2.8. Let $M$ be an embedded triply periodic minimal surface admitting a rotational symmetry $\rho$ with axis of symmetry $x_{3}$. Then the quotient surface $M / \Lambda / \rho$ has genus one.

Proof. We use the Riemann-Hurwitz formula. Notationally, $N^{\prime}$ is $M / \Lambda, N$ is $M / \Lambda / \rho$, and $f$ is the quotient map $f: M / \Lambda \rightarrow M / \Lambda / \rho$. Note that $\gamma \neq 3$, since $f$ is not degree 1. Similarly, if $\gamma=2$, then by Riemann-Hurwitz, $2=n+B / 2$, and so either $n=2$ and the map is unbranched or $n=1$ (which is impossible since rotational symmetries have order at least 2). If $n=2$, then by Corollary 2.6 the map $\rho$ must have 4 fixed points on $M / \Lambda$, implying that $f$ is branched, a contradiction.

Furthermore, the quotient cannot have genus $\gamma=0$, since the height differential $d h$ is invariant under $\rho$; therefore it descends holomorphically to the quotient $M / \Lambda / \rho$. Of course, a surface of genus 0 has no holomorphic differentials, so $\gamma \neq 0$. The only remaining possibility is $\gamma=1$. 
We can use these results to obtain information about the Gauss map of a minimal surface. Let $M / \Lambda$ be an embedded genus three triply periodic minimal surface, and let $\rho \in \operatorname{Aut}(M)$ with $\operatorname{order}(\rho)=2$. Using a rigid motion, we orient $M$ so that the axis of rotational symmetry is the $x_{3}$-axis. By Proposition 2.8, $M / \rho$ is a torus, and so, by Corollary 2.6, $\rho$ has exactly 4 fixed points. The fixed points are precisely those points with vertical normal, and we scale $M$ so that the torus generators are 1 and $\tau$ with $\tau \in \mathbb{C} \cap\{\operatorname{Im} \tau>0\}$. The squared Gauss map $G^{2}$ descends to the quotient torus.

Lemma 2.9. $G^{2}$ has two first-order poles and two first-order zeros.

Proof. Part (1) of Abel's theorem tells us that there must be an equal number of zeros and poles. Suppose, by way of contradiction, that $G^{2}$ had a second-order zero at 0 . Thus $G$ has a zero of at least second order on the genus three surface $M$. Since $d h$ is the lift of $d z$ and since $d z$ has no zeros, $d h$ has zeros of at most first order on the genus three surface in space (locally, the pullback map behaves like $z^{2}$ at a branch point). However, for the metric on $M$ to be nondegenerate and to have no ends, $d h$ must have a zero of at least second order, a contradiction. Therefore, the zeros of $G^{2}$ are of at most first order. The same reasoning holds for the first-order poles.

\section{Parametrization of the gyroid and description of the periods}

The gyroid is the unique embedded member in the associate family of the Schwarz $P$ surface (except for the $\mathrm{D}$ surface, the surface adjoint to the $P$ surface). Therefore, to parametrize the gyroid, we must first understand the $P$ surface. We now give a parametrization of the Schwarz $P$ surface by a convenient Weierstrass representation. Then we describe the gyroid as a specific member of the associate family of the $P$ surface. Finally, we describe the periods of the gyroid in terms of the flat structures.

3.1. The P Surface and tP deformation. The Schwarz $P$ surface (see Figure 3.1) can be constructed in a number of different ways. The approach taken below, while useful for our purposes, is not the most direct parametrization.

The $P$ surface admits an order 2 rotational symmetry $\rho_{2}: \mathbb{R}^{3} \rightarrow \mathbb{R}^{3}$ about the $x_{3}$-axis. Since the rotation is compatible with the action of $\Lambda$ on $\mathbb{R}^{3}, \rho_{2}$ descends to an order 2 symmetry of the quotient surface $P / \Lambda$ (abusing notation, we also call the symmetry induced on the quotient $\rho_{2}$ ). $\rho_{2}$ has four fixed points on $P / \Lambda$ as illustrated in Figure 3.2. (The fixed points of a rotation about a vertical axis are exactly those points with vertical normal. For any genus three triply periodic minimal surface, there are at most four points with vertical normal since the degree of the Gauss map is 2.) The quotient $P / \Lambda / \rho_{2}$ is a (conformal) torus $\mathbb{C} / \Gamma$ (compare 

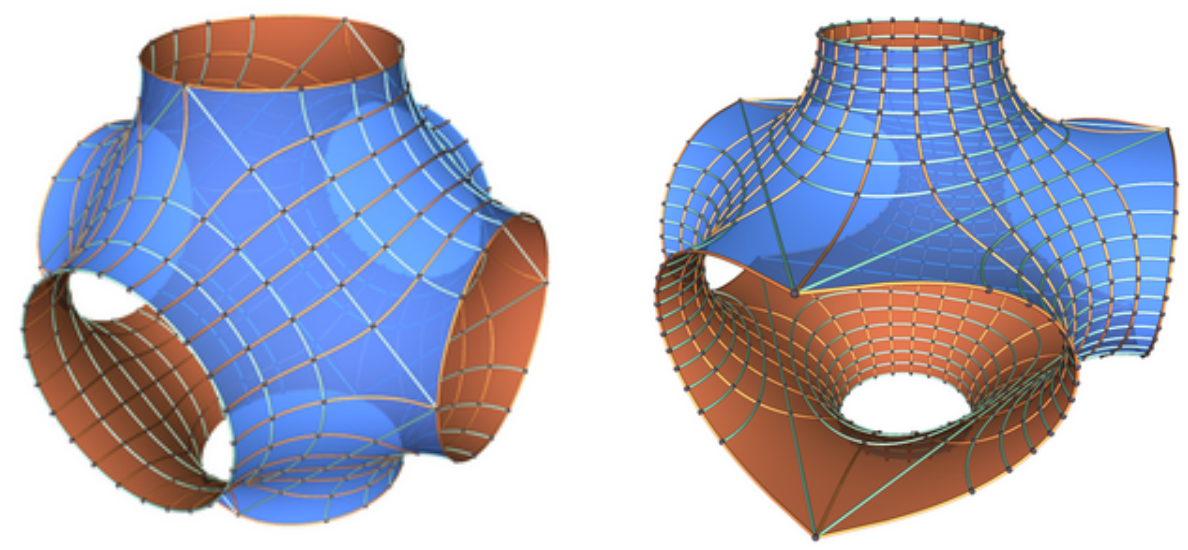

Figure 3.1. Left: A translational fundamental domain of the Schwarz $P$ surface. Right: A fundamental domain of the Schwarz $H$ surface.

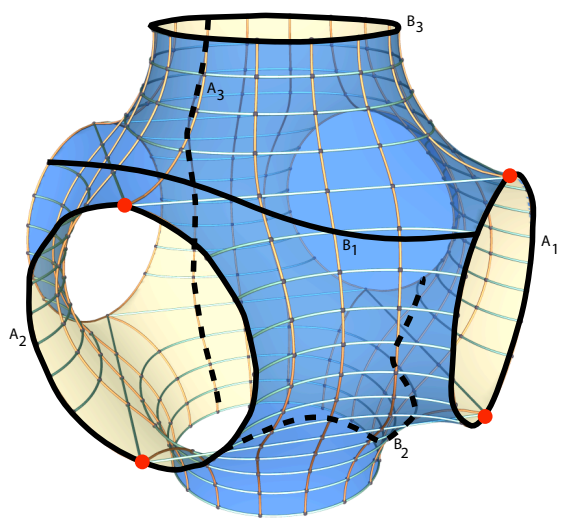

Figure 3.2. $P$ surface with generators for the homology. Fixed points of $\rho_{2}$ are shown in red.

Proposition 2.8 and Corollary 2.6, noticing that $\rho_{2}$ is not the hyperelliptic involution since it fixes only four points).

The lattice $\Lambda$ is the cubical lattice generated by the unit length standard basis vectors $\left\{e_{1}, e_{2}, e_{3}\right\}$. We can restrict the possible conformal structure of the torus $\mathbb{C} / \Gamma$ by considering reflectional symmetries. The $P$ surface admits a reflectional symmetry that also commutes with $\rho_{2}$, namely, the reflection in the plane containing $x_{1}$ and $x_{3}$. Its fixed point set consists of two disjoint totally geodesic curves. Since this reflection commutes with $\rho_{2}$, it descends to the torus $\mathbb{C} / \Gamma$ as a symmetry, which yields two disjoint fixed point sets. The only conformal tori that admit two 
disjoint fixed point sets of a single (orientation reversing) isometry are the rectangular tori (rhombic tori admit orientation reversing isometries with a connected fixed point set). Therefore, $\Gamma$ is generated by $b \in \mathbb{R}$ and $\tau \in i \cdot \mathbb{R}$. Since the conformal structure is unchanged by a dilation in space, we may dilate so that we can take $\Gamma=\langle 1, \tau\rangle$ with $\tau=a i$ for $a \in \mathbb{R}$. (Note that the dilation required to normalize the torus this way may change the lattice $\Lambda$ so that the generators no longer have unit length.) The map $P / \Lambda \rightarrow(P / \Lambda) / \rho^{2}=\mathbb{C} / \Gamma$ is a branched covering map. We can identify (using the aforementioned symmetries) the location (on the torus) of the branch points of this map: branch points corresponding to zeros of $G$ are located at 0 and $\tau / 2$, while branch points corresponding to poles of $G$ are located at $1 / 2$ and $1 / 2+\tau / 2$.

Since the $x_{3}$ coordinate is invariant under $\rho_{2}$, the height differential $d h$ descends holomorphically to the quotient torus as $r e^{i \theta} d z$ for some $r \in \mathbb{R}$ and $0 \leq \theta \leq \pi / 2$ (since $d z$ is, up to a constant multiple, the only holomorphic 1 -form on $\mathbb{C} / \Gamma$ ). Varying $r$ only scales the surface in space, and so $r$ is determined by our requirement that one of the generators of the torus is 1 (we will drop the $r$ for the rest of this work, since scaling is inconsequential to us). $\theta$ is the important Bonnet transformation parameter. For the $P$ surface, $\theta=0$. As noted in Lemma 2.9, the squared Gauss map $G^{2}$ has simple poles and zeros at the branch points.

We can explicitly write the formula for $G^{2}$ using theta functions as

$$
G^{2}(z):=\rho \frac{\theta(z, a i) \theta(z-(a / 2) i, a i)}{\theta(z-1 / 2, a i) \theta(z-(1+a i) / 2, a i)} .
$$

The multivalued function $G$ on $\mathbb{C} / \Gamma$ is obtained by $G(z)=\sqrt{G^{2}(z)}$. The factor $\rho$ is called the Lopez-Ros factor and gives rise to many interesting deformations of minimal surfaces, most of which are not embedded [López and Ros 1991]. If $\rho=r_{1} e^{i \phi}$, varying $\phi$ simply produces a rotation of the minimal surface in space. We will use $\phi$ indirectly to normalize certain quantities. We will also determine the real part $r$ of $\rho$ by a normalization, although varying $r$ is highly destructive: in general, if a surface is embedded for $\rho=\rho_{0}$, modifying $\rho$ will instantly yield a nonimmersed surface (à la the Bonnet transformation). We will determine an appropriate value of $\rho$ for the $P$ surface in Section 3.1.3.

The torus and the branch points are invariant under the symmetry $-i d$; the quotient $S=(\mathbb{C} / \Gamma) /(-i d)$ is a sphere with 4 branch points.

The 1-forms $G d h,(1 / G) d h$, and $d h$ each place a flat structure on the torus which, after taking the quotient with $-i d$, descends to the sphere. We study here the developed image of each flat structure, which we will then use to compute periods. We study each flat structure independently.

3.1.1. $d$ f flat structure for the $P$ surface. Since the $d h$ flat structure descends as $e^{i \theta} d z$, the developed image of the flat structure for the torus is simply the rectangle. 
Consider the "lower half" of the rectangle as a fundamental domain for the action $-i d$, and note the additional identification induced. One can then see directly the sphere $S$. The $d h$ flat structure is, in fact, a rectangle with $\tau / 2$ directly above 0 . This is because there is a horizontal plane of reflection that interchanges the zeros and poles of the Gauss map. The horizontal symmetry curve descends to the quotient torus as a vertical straight line. The reflection only interchanges the branch points if the torus is oriented so that the points corresponding to 0 and $\tau / 2$ in the developed flat structure have the same imaginary part.

3.1.2. Gdh flat structure for the P surface. As noted in the proof of Proposition 2.3 , the order of the zeros and poles of the 1-form $G d h$ produce cone angles on the torus of $3 \pi$ at both 0 and $\tau / 2$ and of $\pi$ at both $1 / 2$ and $1 / 2+\tau / 2$. The involution $-i d$ halves the cone angles in the quotient, so that on the sphere the cone angles are

- a cone point of angle $3 \pi / 2$ at both 0 and $\tau / 2$;

- a cone point of angle $\pi / 2$ at both $1 / 2$ and $1 / 2+\tau / 2$.

The situation is illustrated in Figure 3.3.

Developing the sphere with cone metric induced by $G d h$ gives a hexagon:

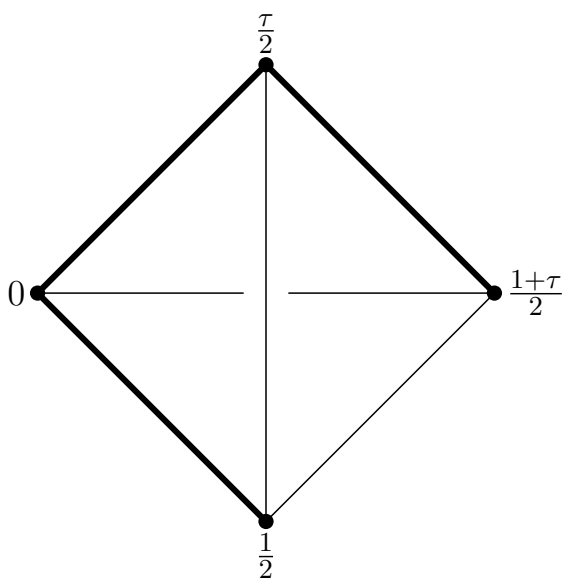

Figure 3.3. A three-dimensional topological picture of the sphere $S$ with flat structure induced by $G d h$. Note that the angles are not drawn correctly. Cone points are visible at the marked vertices. Thick black lines indicate cuts made to develop the sphere (tetrahedron) into the plane. Although this is conformally not the $G d h$ cone metric, this tetrahedron is conformally the development of the $d h$ cone metric (since all points are regular for $d h$ ). 
Lemma 3.1. By cutting along the shortest geodesics on the sphere from $1 / 2$ to 0 , 0 to $\tau / 2$, and from $\tau / 2$ to $1 / 2+\tau / 2$ and developing into the plane, we obtain a hexagon shown in Figure 3.4. The hexagon has the properties

(i) the length of $l_{i}$ is equal to that of $l_{i}^{*}$ for $i=1,2,3$;

(ii) the angle between $l_{1}$ and $l_{2}$ and the angle between $l_{1}^{*}$ and $l_{2}^{*}$ are both $3 \pi / 4$;

(iii) the angle between $l_{1}$ and $l_{1}^{*}$ and the angle between $l_{3}$ and $l_{3}^{*}$ are both $\pi / 2$.

The proof is nearly identical to the more general proof of Lemma 4.1 and is omitted.

At this point, we have not yet determined the value of $a$ for the torus. What is clear is that once $a$ is chosen, the entire $G d h$ flat structure will be fixed. For the moment, we describe the flat structures and study the period problem with this determinacy still unresolved.

3.1.3. $(1 / G)$ dh flat structure for the $P$ surface. $G^{2}(z+1 / 2)$ and $(1 / G)^{2}(z)$ have precisely the same zeros and poles to the same order. By Liouville's theorem, the quotient is constant, since it is holomorphic (with no poles) and doubly periodic. Thus

$$
G^{2}\left(z+\frac{1}{2}\right) /\left(\frac{1}{G}\right)^{2}(z)=r_{1} e^{i \phi_{1}} .
$$

By adjusting the Lopez-Ros parameter, we can ensure that this factor is 1 , and we do that for the $P$ surface. Therefore, the $(1 / G) d h$ flat structure is simply a translation of the (infinite, periodic) $G d h$ flat structure. This is reflected in the outline of the $(1 / G) d h$ flat structure in Figure 3.4.

3.1.4. Compatibility of $G d h,(1 / G) d h$, and $d h$. We have drawn $G d h$ and $d h$ oriented a specific way; namely, the $d h$ flat structure is horizontal, and the $G d h$ flat structure has the line segment $l_{2}$ vertical. We have not yet justified the second of these claims. More generally, any time one prescribes all three data $-G d h$, $(1 / G) d h$, and $d h$ - one has to ensure that $G d h \cdot(1 / G) d h=d h^{2}$. This compatibility is a serious problem when showing the existence of surfaces in general, but the approach taken in Section 4.2 avoids this problem completely.

For the $P$ surface, one can see that this orientation is correct as follows. There is a vertical symmetry plane that interchanges the two zeros of the Gauss map. This reflection descends to the torus, and the symmetry curve is exactly the horizontal line at $y=\operatorname{Im} \tau / 4$ (recall we have fixed a fundamental domain of the torus). After a translation, $d h$ is real on this symmetry curve. Under the flat structure $G d h$, this symmetry curve develops to the line segment from $(0, q / 2)$ to $(2 p, q / 2)$. Again, after a vertical translation, $\int G d h$ is real on this segment. This is also true for $(1 / G) d h$ (the developed flat structure is only a translation of that for $G d h)$. Thus we see that both $G d h$ and $(1 / G) d h$ are real on this segment, and this is compatible 


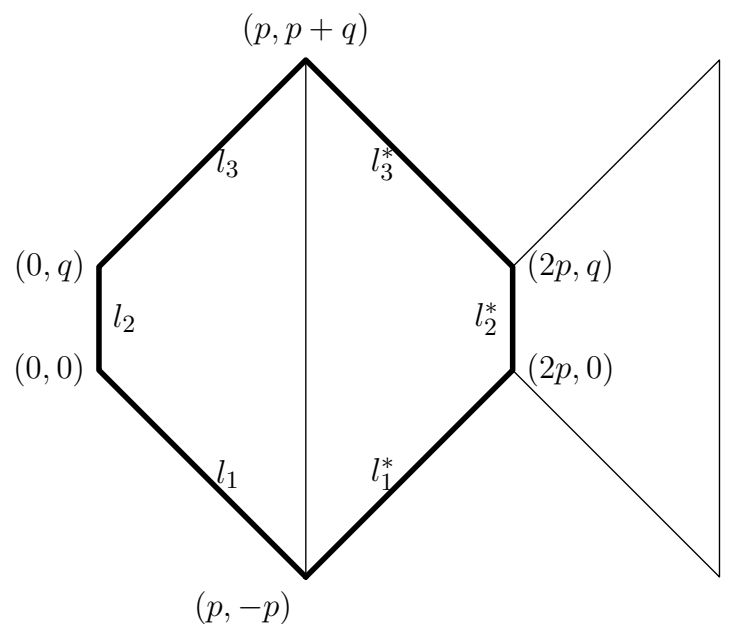

Figure 3.4. The $G d h$ (thick lines) and $(1 / G) d h$ (thin lines) flat structures for the $P$ surface. Labeled vertices are for the $G d h$ flat structure; the corresponding points on $(1 / G) d h$ are obtained by translation by $(-p, p)$.

with $d h$. (The only possible inconsistency is the rotational orientation of $G d h$, so it suffices to check one curve.)

3.2. The period problem for the $P$ surface. The six cycles shown in Figure 3.2 generate the homology $H_{1}(P / \Lambda, \mathbb{Z})$. Figure 3.5 shows these cycles on the 2sheeted branched torus, along with cuts to identify this structure with the surface in space. To compute the periods, we need to compute $\int_{\gamma} G d h$ for each generator $\gamma$ of the homology (and do the same for $(1 / G) d h)$. Since $\int G d h$ is simply the developing map of the $G d h$ flat structure on the torus, we can compute in terms of the cycles' image on the developed flat structure. To calculate the periods, we first obtain the horizontal contribution from the $G d h$ and $(1 / G) d h$ flat structures, for example

$\int_{A_{1}} G d h=(1+i)(p+p i)=2 p \cdot i \quad$ and $\quad \int_{A_{1}} \frac{1}{G} d h=(1-i)(p-p i)=-2 p \cdot i$

The vertical periods are easily read off of the torus as simply the difference in the endpoints of the curves drawn on the torus in Figure 3.2. Recalling that

$$
P(\gamma)=\left(\operatorname{Re}\left(-\int_{\gamma} G d h+\overline{\int_{\gamma} 1 / G d h}\right), \operatorname{Im}\left(-\int_{\gamma} G d h+\overline{\int_{\gamma} 1 / G d h}\right), \operatorname{Re} \int_{\gamma} d h\right),
$$



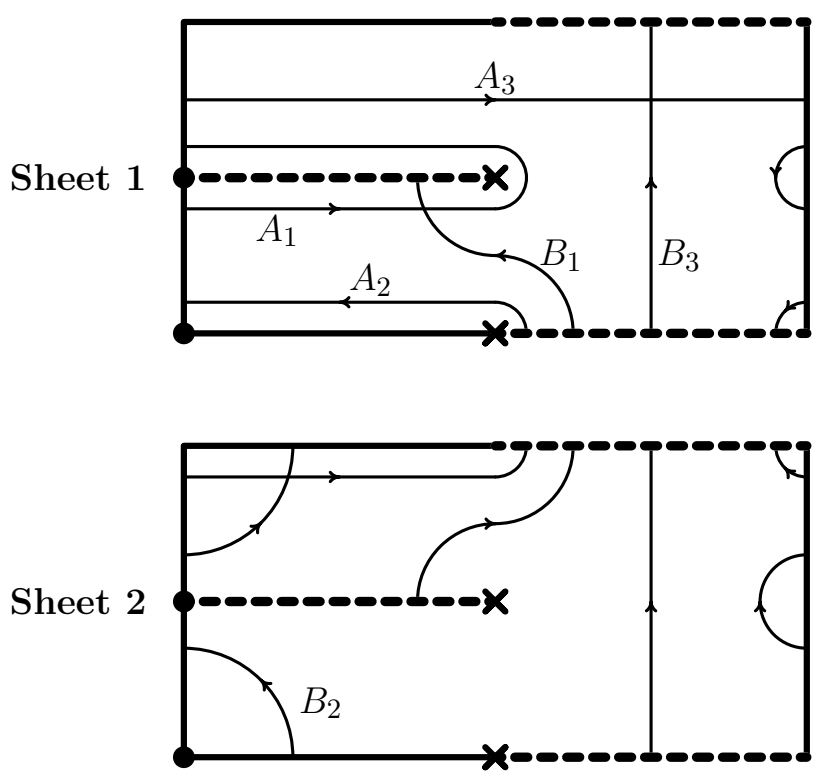

Figure 3.5. The conformal model of the $P$ surface showing the homology generators. Cuts to reconstruct the surface by gluing are shown by dashed lines. Branch points corresponding to zeros of the Gauss map are shown by solid dots, while poles are indicated by an $\mathrm{X}$.

we write

$$
\begin{array}{ll}
P\left(A_{1}\right)=(0,0,0), & P\left(B_{1}\right)=(2(p+q),-2(p+q), 0), \\
P\left(A_{2}\right)=(0,0,0), & P\left(B_{2}\right)=(-2(p+q),-2(p+q), 0), \\
P\left(A_{3}\right)=(0,0,1), & P\left(B_{3}\right)=(0,0,0) .
\end{array}
$$

(This last horizontal period is zero due to the 2-fold symmetry of this curve. Since the cycle continues onto both sheets, we develop from $p_{1}$ to $p_{4}$, then rotate $180^{\circ}$ (to get on the other sheet), then develop the same length again. This causes the horizontal period for $B_{3}$ to vanish.) It is immediately clear that these periods generate a 3-dimensional lattice $\Lambda$ for all values of $p$ and $q$. In other words, the period problem is solved no matter what the actual lengths of the segments in the developed flat structure are. Thus any value of a (and therefore, any quotient torus) solves the period problem. 
While we have phrased this section as if we were describing the $P$ surface, what we have actually seen is that there is a family of immersed triply periodic minimal surfaces that contains the $P$ surface.

Theorem 3.2. There exists a continuous family of embedded triply periodic minimal surfaces of genus three that contains the P surface (the tP family). Each member of the family admits an order 2 rotational symmetry and has a horizontal reflective symmetry plane.

This is a consequence of the fact that all rectangular tori (with the given Weierstrass data) solve the period problem. (Embeddedness follows from Proposition 4.6.) Note that all of these surfaces are in the Meeks family.

We will call this family of minimal surfaces the $t P$ family (Figure 3.6). Note that the limit $\tau \rightarrow 0$ looks like a pair of parallel planes joined with small catenoidal necks. The limit $\tau \rightarrow \infty$ looks like a pair of perpendicular planes that are desingularized along the intersection by adding handles (like the singly periodic Scherk surface). See [Traizet 2008] for interesting results related to these limiting surfaces.

3.3. The gyroid. We are finally ready to describe the gyroid minimal surface. Schoen [1970] describes a surface that is associate to the $P$ and $D$ surfaces and is embedded. Let $(X, G, d h)$ be the Weierstrass data describing the $P$ surface (see Section 3.1). Recall that a surface is called associate to $(X, G, d h)$ if its Weierstrass data is $\left(X, G, e^{i \theta} d h\right)$. For a single value of $\theta$, this associate surface is an embedded minimal surface, which Schoen called the gyroid. In his description of the gyroid, Schoen estimated $\theta \approx 38.0147740^{\circ}$. That this value of $\theta$ along with $\theta=0$ ( $P$ surface) and $\theta=\pi / 2$ (D surface) are the only values that produce an embedded minimal surface is something of a curiosity. In [Große-Brauckmann and Wohlgemuth 1996], the gyroid is described geometrically as follows. The angle of association for the gyroid is such that the vertical period of $B_{3}$ must be twice that of $A_{3}$. Since the $B_{3}$ curve continues on both sheets of the torus, we need the images of the curves on a single sheet of the developed image of the $d h$ flat structure of the torus to have equal real part. This is equivalent to choosing $\theta$ so that the rotated $d h$ flat structure places the point $1+\tau$ directly above 0 in the developed image (see Figure 3.7). Therefore $\theta=\operatorname{arccot} \operatorname{Im} \tau$.

Unfortunately the value of $\tau$ that gives the standard, most symmetric $P$ surface still must be determined by an elliptic integral. In other words, if $(X, G, d h)$ is any member of the $t P$ family, then $\left(X, G, e^{i \theta} d h\right)$ is an embedded surface only if $\theta=0$, $\theta=\pi / 2$, or $(X, G, d h)$ describe the most symmetric (what we call the "standard") $P$ surface. As an unfortunate consequence of this fact, we see that varying $\tau \in i \cdot \mathbb{R}$ is not enough to yield a family of gyroids - we must consider $\tau \in \mathbb{C}$. 


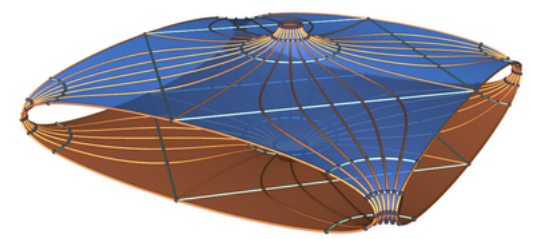

$$
\tau=0.24
$$

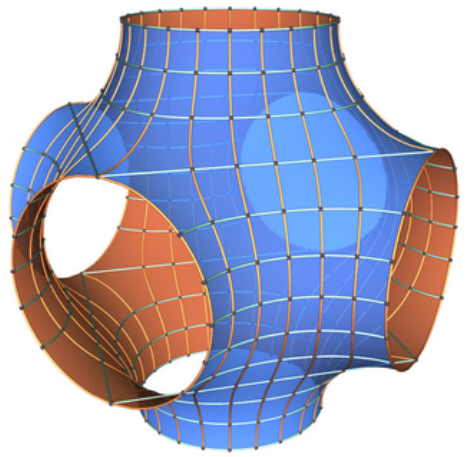

$$
\tau=0.781
$$

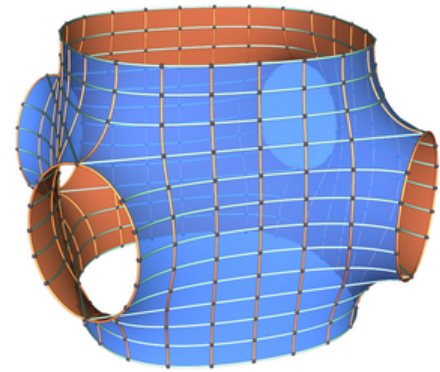

$$
\tau=1.70
$$

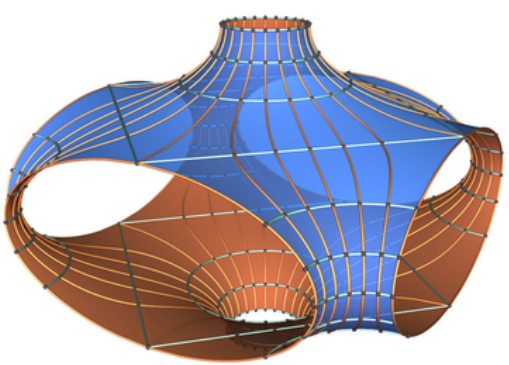

$\tau=0.40$

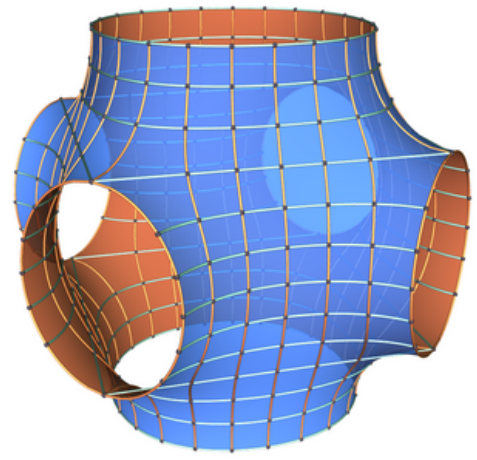

$\tau=1.10$

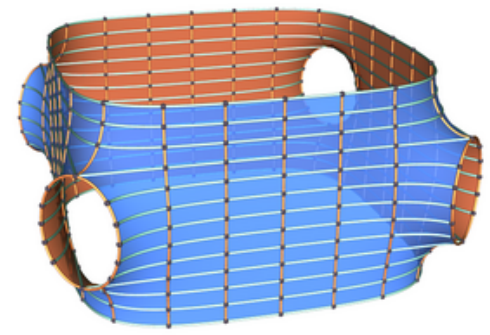

$$
\tau=3.00
$$

Figure 3.6. The $t P$ deformation of the $P$ surface, for different values of $\tau$.

A quick computation using the resulting flat structures gives the following periods for the gyroid:

$$
\begin{array}{ll}
P\left(A_{1}\right)=(1,0,0), & P\left(B_{1}\right)=(1,0,-1), \\
P\left(A_{2}\right)=(1,0,0), & P\left(B_{2}\right)=(-1,0,-1), \\
P\left(A_{3}\right)=(0,1,1), & P\left(B_{3}\right)=(0,0,2) .
\end{array}
$$




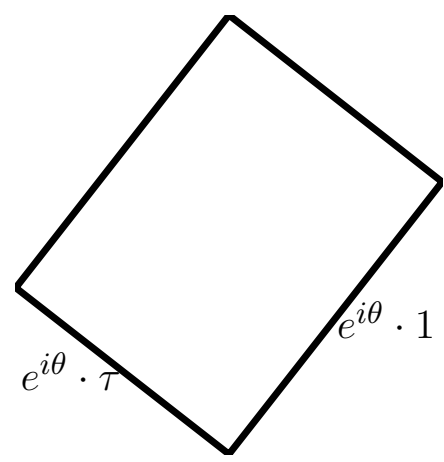

Figure 3.7. The alignment of the $d h$ flat structure for the gyroid. The alignment of the $d h$ flat structure for the gyroid.

We have gone through a fairly complicated set of gymnastics to show that the gyroid is even immersed, but this complicated method does nicely set up the period problem for Section 4.2, where we prove the existence of two families of gyroids. There is, however, a much easier way to see that the gyroid is immersed. Instead of considering the flat structures induced by $G d h,(1 / G) d h$, and $d h$, consider instead the Weierstrass 1 -forms $\omega_{1}, \omega_{2}$, and $\omega_{3}=d h$. The standard $P$ surface admits an order 3 rotational symmetry that interchanges each of the coordinate axes; the action of this rotation on the space of holomorphic forms permutes the 1-forms $\omega_{i}$. The flat structures, therefore, are all congruent, and all of the periods can be expressed in terms of these 1 -forms. Since the associate family parameter $\theta$ solves the vertical period problem and since the flat structures of these forms are all congruent, the period problem is completely solved. Even though this brief proof easily shows that the gyroid is immersed, this technique seems to fail miserably at achieving a family of gyroids. As soon as we lose the symmetry of the standard $P$ surface, the technique is no longer useful.

\section{Proof of main theorem}

In this section, we prove Theorem 1.2, which says there exists a family of gyroids that preserve an order 2 rotational symmetry.

4.1. Sketch of the proof. First, define a moduli space of polygons $\mathscr{H}(G)$ that solve the horizontal period problem. That is, suppose $X$ is a Riemann surface constructed as the branched (double) cover of a torus $T$, a Gauss map $G$, and a height differential $d h$ so that the developed image of the torus $T$ with cone metric induced by $G d h$ is in $\mathscr{H}(G)$. Then the horizontal periods will lie in a lattice. Also, the generators of the lattice will be the periods of the same cycles that generate the lattice for the gyroid. (In fact, our tori will have the property that they are invariant under $-i d$, 
so we only develop $T /-i d$.) Note that the lattice will not be constant throughout the deformation. Since the horizontal period problem requires knowledge about not only $G d h$ but also $(1 / G) d h$, we impose a normalization so that the $(1 / G) d h$ flat structure is a translate of the flat structure developed by $G d h$.

Second, define a moduli space of polygons $\mathscr{V}(G)$ that solve the vertical period problem. Since the conformal model of a Riemann surface is, in our case, always the (two or three)-fold cover of a torus, the vertical moduli space will always consist of parallelograms. The critical issue here will be the orientation of the developed image of the parallelograms. (In fact, orientation of the developed flat structure is also the critical issue for $\mathscr{H}(G)$.)

Then, we show that there exists a set of Weierstrass data $\left\{X_{\eta}, G_{\eta}, d h_{\eta}\right\}$ for $\eta \in \mathbb{R}$ such that the developed image of the torus $T_{\eta}$ under the flat structure induced by $G d h$ is in $\mathscr{H}(G)$ and that induced by $d h$ is in $\mathscr{V}(G)$. This shows that both the horizontal and the vertical period problems can be solved simultaneously by a family of Weierstrass data. To accomplish this, define a continuous function $h: \mathbb{C} \rightarrow \mathbb{R}$ with the property that $h(\tau)=0$ implies that there exists $\theta(\tau)$ such that the $G d h$ flat structure (respectively the $d h$ flat structure ) will be in $\mathscr{H}(G)$ (respectively $\mathscr{V}(G)$ ) provided that $d h=e^{i \theta}$. The Gauss map will be determined by the conformal structure of the torus and the normalization requiring that the $G d h$ and $(1 / G) d h$ flat structures are translates. We then show that $h^{-1}(0)$ contains a curve and that this curve contains the value $\tau$ that determines the standard gyroid. This guarantees the existence of a continuous family of immersed triply periodic minimal surfaces that contains the gyroid. To study the zero set of $h$, we use that we can compute $h$ more or less explicitly for rectangular tori. Also, we can compute $h$ for $\tau=n+y i$ for $n \in \mathbb{Z}$ by studying the effect of twists on the torus and the flat structures. This allows us to compute sufficiently many values to use an intermediate value argument.

Finally, we show that the surfaces obtained in this way are embedded (and not just immersed), a consequence of the maximum principal for minimal surfaces; see the survey [López and Martín 1999]. We separate the embeddedness portion of the proof into the more general Proposition 4.6.

In Section 4.2, we set up the moduli spaces $\mathscr{H}(G)$ and $\mathscr{V}(G)$. In Section 4.3, we prove the remaining statements. We do this in detail for the $t G$ family. For the families $r G$ and $r L$, we construct the moduli spaces in Section 5.

4.2. Horizontal and vertical moduli spaces for the tG family. In the most general setting, it is not possible to split the period problem into vertical and horizontal components. In our case however, we are considering surfaces that are invariant under rotation. Therefore, since the height is invariant under this rotation, the height differential $d h$ establishes a consistent $x_{3}$ direction that is invariant throughout the 
family. Therefore, the lattice $\Lambda$ is a product $\mathbb{Z} \times \Lambda_{1}$, and so we can split the period problem into two parts. We need to show that there is a single vertical period, and that the horizontal periods lie in a two-dimensional $\mathbb{Z}$-lattice.

4.2.1. Definition of $\mathscr{V}(G)$ and calculation of the vertical periods. In this subsection we describe the conformal models of the surfaces we wish to construct. Recall that the underlying Riemann surface structure for the gyroid was a 2-fold branched cover of a rectangular torus that parametrized the $P$ surface (here we are referring to the most symmetric $P$ surface).

Denote by $\widetilde{\mathscr{V}(G)}$ the space of marked parallelograms in $\mathbb{C}$ up to equivalence by translations (we consider marked parallelograms to distinguish the cone point 0 ). Notice that if $\Gamma=\langle 1, \tau\rangle$ is a $\mathbb{Z}$-lattice in $\mathbb{C}$, the torus $\mathbb{C} / \Gamma$, once equipped with the flat structure induced by $e^{i \theta} d z$, develops to an element of $T \in \widetilde{\mathscr{V}(G)}$. If $M$ is a triply periodic minimal surface with symmetry $\rho_{2}$ such that $M / \Lambda / \rho_{2}=\mathbb{C} / \Gamma$, and if we develop generators of the homology $H_{1}(M, \mathbb{Z})$ onto $T$, then $M$ is immersed only if both the horizontal and the vertical period problems are solved. The period problem is in general not solved if $\mathbb{C} / \Gamma$ develops into $\widetilde{\mathscr{V}(G)}$.

We will now define a subset of $\widetilde{\mathscr{V}(G)}$ that does solve the vertical period problem. There are generally many such subsets, but we seek a deformation of the gyroid. Recall (see Figure 3.7) that the gyroid's $d h$ flat structure for the torus satisfies $\operatorname{Re} e^{i \theta}=-\operatorname{Re} e^{i \theta} \tau$. With this motivation, we define

$$
\mathscr{V}(G)=\left\{\left(\omega_{1}, \omega_{2}\right) \in \mathbb{C} \times \mathbb{C}|| \omega_{1} \mid=1 \text { and } \operatorname{Re} \omega_{1}=-\operatorname{Re} \omega_{2}\right\} .
$$

Developing the cycles shown in Figure 3.2 onto this flat structure, one easily sees that the vertical period problem is solved. Using the notation of the cycles from Figure 3.5, the vertical periods are

$$
\begin{array}{ll}
P\left(A_{1}\right)=(--,--, 0), & P\left(B_{1}\right)=\left(--,--,-\operatorname{Re} \omega_{1}\right), \\
P\left(A_{2}\right)=(--,--, 0), & P\left(B_{2}\right)=\left(--,--,-\operatorname{Re} \omega_{1}\right), \\
P\left(A_{3}\right)=\left(--,--, \operatorname{Re} \omega_{1}\right), & P\left(B_{3}\right)=\left(--,--, 2 \operatorname{Re} \omega_{1}\right) .
\end{array}
$$

4.2.2. Definition of $\mathscr{H}(G)$ and calculation of the horizontal periods. Suppose that $M$ is any immersed, genus three, triply periodic minimal surface that has as a conformal model a two-fold branched cover of a generic torus $\mathbb{C} / \Gamma$. Without loss of generality we write $\Gamma=\langle 1, \tau\rangle$. Suppose further that the square of the Gauss map descends to $\mathbb{C} / \Gamma$ and has simple poles at $1 / 2$ and $1 / 2+\tau / 2$ and simple zeros at 0 and $\tau / 2$. (This is the case for the gyroid, except that the torus is rectangular.) The quotient $S=\mathbb{C} / \Gamma /(-i d)$ is a sphere, and $G d h$ again induces a cone metric on $S$. Under this cone metric, the sphere is a tetrahedron with two vertex angles $3 \pi / 2$ and two vertex angles $\pi / 2$. The developed image of this sphere has a particularly nice parametrization: 


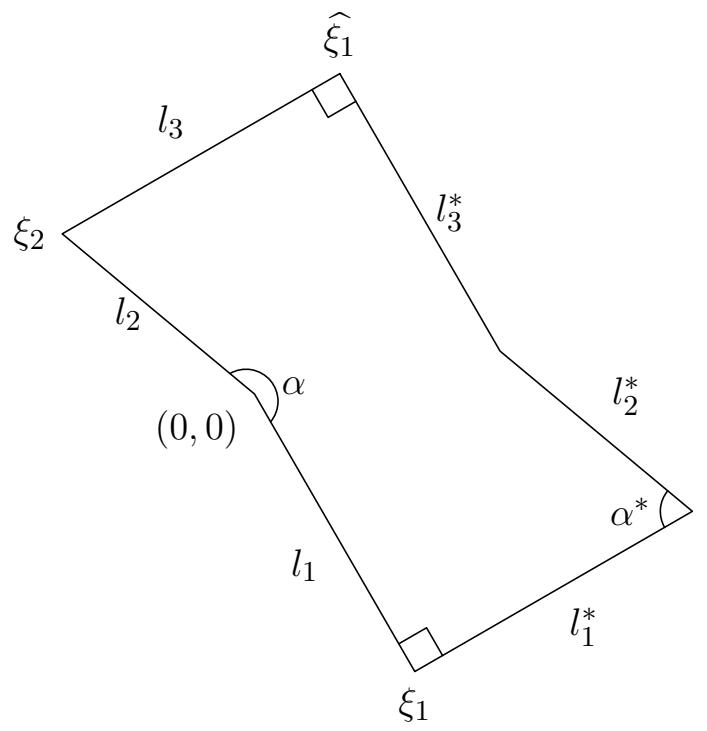

Figure 4.1. A generic member of the $\widetilde{\mathscr{H}(G)}$ moduli space.

Lemma 4.1. For any torus $\mathbb{C} / \Gamma$ with $\Gamma=\langle 1, \tau\rangle$ the cone metric $G d h$ descends to $S$. By cutting along shortest geodesics on $S$ from $1 / 2$ to 0,0 to $\tau / 2$, and from $\tau / 2$ to $1 / 2+\tau / 2$, we obtain a hexagon; see Figure 4.1. The hexagon has the properties

(i) the length of $l_{i}$ is equal to that of $l_{i}^{*}$ for $i=1,2,3$;

(ii) $l_{2}$ is parallel to $l_{2}^{*}$;

(iii) the angle between $l_{1}$ and $l_{1}^{*}$ and the angle between $l_{3}$ and $l_{3}^{*}$ are both $\pi / 2$.

We can parametrize the space of possible hexagons by $\xi_{1}, \xi_{2} \in \mathbb{C}$ as shown in Figure 4.1.

We call the space of all hexagons satisfying the conditions of Lemma $4.1 \widetilde{\mathscr{H}(G)}$. Proof. Since $\mathbb{C} / \Gamma /(-i d)$ is a sphere and since $-i d$ fixes the branch points of $\mathbb{C} / \Gamma$, the flat structure induced by $G d h$ makes $S$ a tetrahedron with cone angle $3 \pi / 2$ at 0 and $\tau / 2$ and with cone angle $\pi / 2$ at $1 / 2$ and $(1+\tau / 2)$. By making the indicated cuts, we obtain a hexagon with sides $l_{1}, l_{2}, l_{3}, l_{1}^{*}, l_{2}^{*}$, and $l_{3}^{*}$. We denote the points in the developed image corresponding to $1 / 2,0, \tau / 2$, and $(1+\tau / 2) \in \mathbb{C} / \Gamma$ by $p_{1}, p_{2}$, $p_{3}$, and $p_{4}$, respectively. By making a translation, we arrange so that $p_{2}=0 \in \mathbb{C}$. Each $l_{i}$ was identified with $l_{i}^{*}$ before the cutting; therefore, the length of $l_{i}$ is equal to $l_{I}^{*}$. Also, since there is a $\pi / 2$ cone angle at $1 / 2$, the angle between lines $l_{1}$ and $l_{1}^{*}$ must be $\pi / 2$ (and similarly for $l_{3}$ ).

Let $\alpha$ denote the angle between $l_{1}$ and $l_{2}$, and let $\alpha^{*}$ denote the angle between $l_{1}^{*}$ and $l_{2}^{*}$. The cone angle at 0 is $3 \pi / 2$, therefore, since both $p_{2}$ and $p_{2}^{*}$ correspond to 
$0 \in T$, the sum $\alpha+\alpha^{*}=3 \pi / 2$. One can see this by developing a small circle about 0 and noting that in the developed image we must obtain an arc that subtends an angle of $3 \pi / 2$.

To understand the horizontal periods, we again adjust $\rho$, if necessary, to normalize the $(1 / G) d h$ flat structure as in Section 3.1.3 so that the developed flat structure for $(1 / G) d h$ is simply a translate of that for $G d h ; \rho$ is uniquely determined by this normalization. Then in terms of these flat structures, we compute the periods of the six generators of $H_{1}(M, Z)$ and tabulate them:

\begin{tabular}{lll}
\hline$X$ & $\int_{X} G d h$ & $\int_{X}(1 / G) d h$ \\
\hline$A_{1}$ & $(1+i)\left(\widehat{\xi_{1}}-\xi_{2}\right)$ & $(1-i)\left(\xi_{2}+i w-\widehat{\xi_{1}}+\xi_{1}\right)$ \\
$B_{1}$ & $(1+i)\left(\widehat{\xi_{1}}-\xi_{1}\right)$ & $(1-i) \xi_{2}$ \\
$A_{2}$ & $(i-1) \xi_{1}$ & $(1-i) \xi_{1}$ \\
$B_{2}$ & $(1-i) \xi_{2}$ & $(1+i)\left(\widehat{\xi}_{1}-\xi_{1}\right)$ \\
$A_{3}$ & $(-1-i) \xi_{1}$ & $(-1-i) \xi_{1}$ \\
$B_{3}$ & $2\left(\widehat{\xi_{1}}-\xi_{1}\right)$ & $2 \xi_{2}$ \\
\hline
\end{tabular}

The notation $\widehat{\xi}$ is the complex number corresponding to $p_{4}$ (see Lemma 4.1), that is,

$$
\widehat{\xi_{1}}=-\xi_{1}+\xi_{2}+\frac{(2+2 i) \xi_{1}^{2} \bar{\xi}_{1}}{2\left|\xi_{1}\right|^{2}} .
$$

To simplify the calculations, we make the change of variables

$$
a=2\left(\operatorname{Re} \xi_{1}+\operatorname{Im} \xi_{2}\right) \quad \text { and } \quad b=2\left(\operatorname{Im} \xi_{1}-\operatorname{Im} \xi_{2}\right) .
$$

One can then compute the horizontal periods to be

$$
\begin{array}{lll}
P_{A_{1}}=(a+b, 0,--), & P_{A_{2}}=(a+b, 0,--), & P_{A_{3}}=(0, a+b,--), \\
P_{B_{1}}=(a, b,--), & P_{B_{2}}=(-a, b,--), & P_{B_{3}}=(0,0,--) .
\end{array}
$$

Notice that when $b=0$, the period problem is solved. In particular, when $b=0$ the periods coincide with those of the gyroid; see Equation (3-1)) Recall that $b=$ $2\left(\operatorname{Im} \xi_{1}-\operatorname{Im} \xi_{2}\right)$; define

$$
\mathscr{H}(G)=\left\{\left(\xi_{1}, \xi_{2}\right) \in \widetilde{\mathscr{H}(G)} \mid \operatorname{Im} \xi_{1}=\operatorname{Im} \xi_{2}\right\} .
$$

Then every flat structure in $\mathscr{H}(G)$ solves the horizontal period condition, and does so with the same relations among the generating curves as for the gyroid. Figure 4.2 shows a typical member of $\mathscr{H}(G)$.

Of course, there are other choices for $a$ and $b$ that also solve the horizontal period problem. We make this choice because we want the family to contain the gyroid. The choice $a=-b$, for example, would yield the $t P$ family of Section 3.1. 


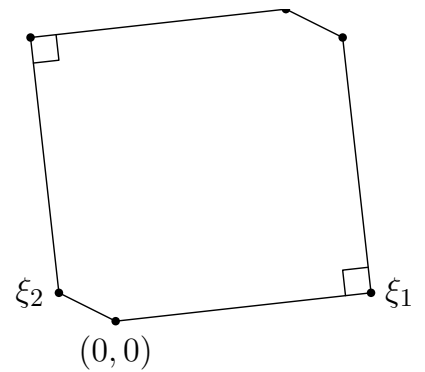

Figure 4.2. A generic member of the $\mathscr{H}(G)$ moduli space.

We have shown that if $(X, G, d h)$ has as flat structures members of $\mathscr{H}(G)$ and $\mathscr{V}(G)$, then the period problem is solved. Certainly the Weierstrass data for the gyroid do solve the period problem. It remains to find a 1-parameter family of such data. We will then show that the surfaces are all embedded.

4.3. Proof of the $t \boldsymbol{G}$ family. To prove the existence of the $t G$ family, our first task is to show that there exists a family of Weierstrass data $(X, G, d h)$ with $X$ the double branched cover of a torus such that the developed image of $\mathbb{C} / \Gamma /(-i d)$ under $G d h$ is in $\mathscr{H}(G)$ and such that the developed image of $\mathbb{C} / \Gamma /(-i d)$ under $d h$ is in $\mathscr{V}(G)$. This will show that the period problem is solved.

Let $\Gamma=\langle 1, \tau\rangle$. Define $d h=d z$ on $\mathbb{C} / \Gamma$. Define $X_{\tau}$ to be the Riemann surface obtained from the double cover of $\mathbb{C} / \Gamma$, with branch points at $0,1 / 2, \tau / 2$, and $1 / 2+\tau / 2$ and with branch cuts as shown in Figure 3.5. The square of the Gauss map will be well defined on $\mathbb{C} / \Gamma$ as the unique meromorphic function with zeros at 0 and $\tau / 2$ and poles at $1 / 2$ and $1 / 2+\tau / 2$, up to a complex multiple $\rho$. Define $\rho$ so that the $G d h$ and $(1 / G) d h$ flat structures are normalized as in Section 3.1.3, that is, so that they are translates.

For each choice $\tau \in \mathbb{C}$, this data describes a minimal surface.

Definition 4.2. The vertical relative turning angle $\theta_{\mathscr{V}}(\tau)$ is

$$
\theta_{\mathscr{V}}(\tau):=\pi / 2-\arg (1+\tau) .
$$

This is precisely the angle by which the $d h$ flat structure fails to be in $\mathscr{V}(G)$.

The horizontal relative turning angle $\theta_{\mathscr{H}}(\tau)$ is the angle by which the $G d h$ flat structure must be rotated so that it satisfies $\operatorname{Im} \xi_{1}=\operatorname{Im} \xi_{2}$.

If $\theta_{V}(\tau)=\theta_{\mathscr{H}}(\tau)$, then we could define $d h=e^{i \theta_{V}(\tau)} d z=e^{i \theta_{\mathscr{H}}(\tau)} d z$. The definition of horizontal and vertical turning angle ensures that $(X, G, d h)$ solves the horizontal and vertical period problem. Define $b(\tau):=\theta_{\mathscr{H}}(\tau)-\theta_{\mathscr{V}}(\tau)$. The period problem is solved exactly on the zero set of $b$. Let $\tau_{G}$ denote the value of $\tau$ which yields the gyroid; $\tau_{G} \approx 0.781 i$. 


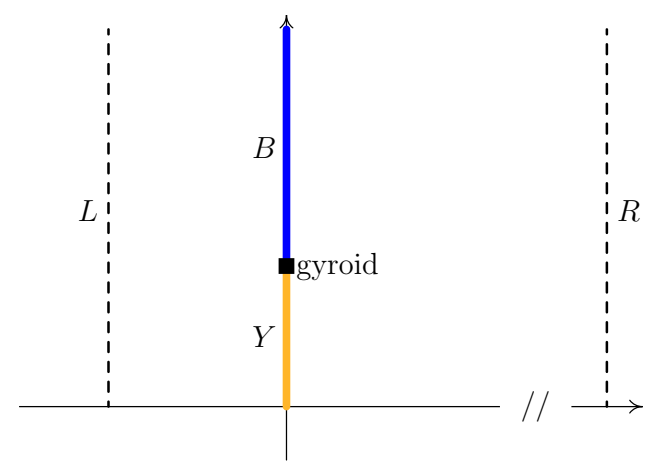

Figure 4.3. $b<0$ on $Y$ and $b>0$ on $B$, so the zero set contains a curve separating $B$ and $Y$ (it must pass through the value that yields the gyroid).

Our goal is to understand the zero set of $b$. Note that when $\tau \in i \mathbb{R}$, the resulting torus is rectangular. On rectangular tori, it is possible to explicitly develop the cone metric $G d h$ into $\mathbb{C}$ by integrating the Gauss map (recall that the Gauss map can be explicitly given in terms of theta functions) and to therefore understand $b$. On a generic, nonrectangular torus explicit computation is not possible, since the edges of a fundamental domain are no longer fixed point sets of an isometry and thus are not totally geodesic. It is no longer the case that these edges of a fundamental domain develop, under integration, to the shortest geodesic between cone points of the tetrahedron.

Next, as in Figure 4.3, consider the half plane, with the $y$-axis divided into two segments $B$ and $Y$, where $Y=\left\{(0, y) \mid \operatorname{Im}(y)<\operatorname{Im} \tau_{G}\right\}$.

Lemma 4.3. $b>0$ on $L$ and $R$, where $L$ is a vertical line $x=-1$ and $R$ is $a$ vertical line $x=n$ for $n \in \mathbb{Z}$ sufficiently large.

Proof. The quotient sphere of the torus generated by $(1,1+\tau)$ is related to the sphere obtained from the torus generated by $(1, \tau)$ by performing a Dehn twist on the cycle $A_{1}$. To understand the effect of the Dehn twist on the $G d h$ flat structure, note that after the twist, $\xi_{2}, \xi_{1}+\hat{\xi}_{1}$, and $\hat{\xi}_{1}$ are translated by $\xi_{1}+\hat{\xi}_{1}-\xi_{2}$, while the remaining vertices of the developed flat structure are fixed.

We can compute the $G d h$ flat structure explicitly in the case of normalized rectangular tori and flat structures: for all rectangular tori, the angle between $l_{1}$ and $l_{2}$ is $3 \pi / 4$ and when normalized (recall that this requires that the $G d h$ and $(1 / G) d h$ flat structures are aligned), the segment $l_{2}$ is vertical with $\operatorname{Im} \xi_{2}>0$. This last is a consequence of the symmetries of rectangular tori; see Section 3.1. 

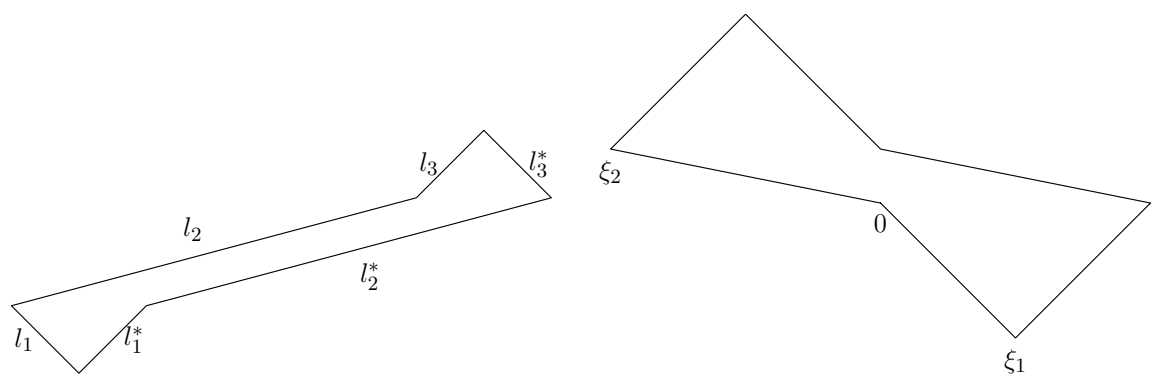

Figure 4.4. Left: The $G d h$ flat structure after many positive Dehn twists. Right: A "rectangular torus" $G d h$ flat structure after applying a single negative Dehn twist.

After a large number of positively oriented Dehn twists, we see a $G d h$ flat structure as in Figure 4.4. Therefore $\theta_{\mathscr{H}}(n+\tau) \approx \pi$ for large $n \in \mathbb{Z}$. This value is larger than $\theta_{\mathcal{V}}(n+\tau) \approx \pi / 2$. Thus $b \approx \pi / 2>0$ on $R$.

Showing that $b>0$ on $L$ is similar other case. After applying a single negatively oriented Dehn twist, $\theta_{\mathscr{H}}$ remains positive ( $\theta_{\mathscr{H}}$ is always positive), but $\theta_{\mathscr{V}}(-1+c i)=$ 0 for all $c \in i \mathbb{R}$. Therefore $b>0$ on $L$, since a negatively oriented Dehn twist shifts the "top cone" of the $G d h$ flat structure; see Figure 4.4.

\section{Lemma 4.4. $b>0$ on $B$ and $Y$.}

Proof. We will show that both $\theta_{\mathscr{V}}$ and $\theta_{\mathscr{H}}$ are monotone - decreasing and increasing, respectively - as $\operatorname{Im} \tau$ increases. This implies that the $b$ has at most 1 zero. Of course, we know that a zero occurs at $\tau_{G}$, yielding the gyroid.

Fortunately, we are able to explicitly calculate $\theta_{\mathscr{V}}$ as $\theta_{\mathscr{V}}(\tau)=\operatorname{arccot}(\operatorname{Im} \tau)$. This function is clearly monotone decreasing in $\operatorname{Im} \tau$.

The situation for the horizontal turning angle is not as simple. First, for all $\tau \in i \mathbb{R}$, the $G d h$ flat structure is normalized in the same orientation as it is for the gyroid, that is, the straight line segment $\lambda$ from the developed image of 0 to the developed image of 1 is horizontal. To see this, observe that in the rectangular case, there is a vertical symmetry curve in space that translates, on the torus, to a horizontal curve (straight line) connecting 0 and 1 . The endpoints have no horizontal displacement, and so must be conjugate in the $G d h$ and $(1 / G) d h$ flat structures. Since all surfaces in the $t P$ family share this symmetry, the line $\lambda$ must be horizontal.

Since for all $\tau \in i \mathbb{R}$ the normalized $G d h$ flat structure is aligned so that $\lambda$ is horizontal when the angle of association is 0 , the relative turning angle in the rectangular case is computed in terms of the ratio of $\left|l_{1}\right|$ to $\left|l_{2}\right|$; precisely,

$$
\theta_{\mathscr{H}}(\tau)=\pi-\arg \left(\left(\left|l_{2}\right| /\left|l_{1}\right|\right) i-e^{-i \pi / 4}\right) .
$$




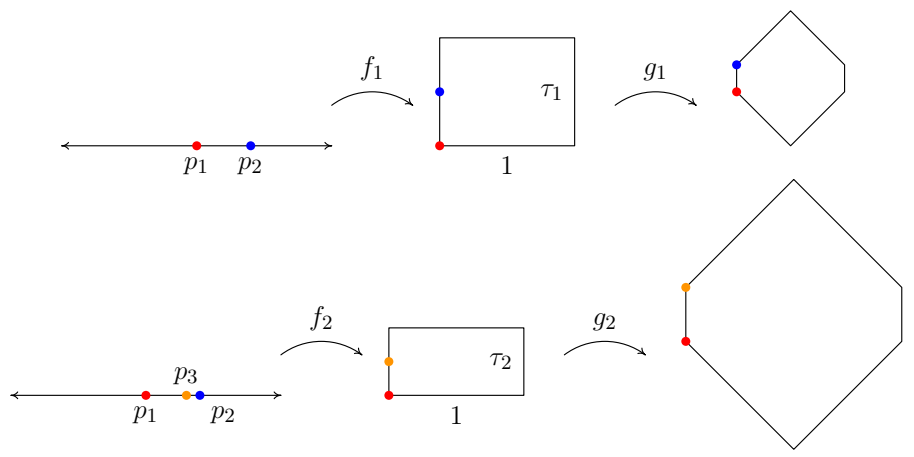

Figure 4.5. Two maps from the upper half plane to a hexagon.

Therefore $\theta \mathscr{H}(\tau)$ increases as the ratio $\left|l_{2}\right| /\left|l_{1}\right|$ increases. We now show that $\left|l_{2}\right| /\left|l_{1}\right|$ is monotone in $\operatorname{Im} \tau$.

Suppose that there exist $\tau_{1}, \tau_{2} \in i \mathbb{R}$ such that $\left|l_{2}^{\tau_{1}}\right| /\left|l_{1}^{\tau_{1}}\right|=\left|l_{2}^{\tau_{2}}\right| /\left|l_{1}^{\tau_{2}}\right|$. Because of the restrictions on the flat structures imposed by the rectangular torus (see Lemma 3.1), this implies that the $G d h$ flat structures are dilations of each other. Call the developing map from the torus $\mathbb{C} /\left\langle 1, \tau_{i}\right\rangle$ to the plane (yielding a hexagon) $g_{i}$. The Schwarz-Christoffel maps $f_{i}$ map the upper half plane to the tori $\mathbb{C} /\left\langle 1, \tau_{i}\right\rangle$. Composing gives two maps from the upper half plane to similar hexagons; see Figure 4.5 .

Thus $g_{1} \circ f_{1}$ and $\frac{1}{2} \cdot g_{2} \circ f_{2}$ are two maps from the upper half plane to the same hexagon. By the Riemann mapping theorem, there is a unique such map up to Möbius transformation, which is however fixed because both maps send $p_{1}$ to the same point. Since $g_{1} \circ f_{1}\left(p_{2}\right)=\frac{1}{2} \cdot g_{2} \circ f_{2}\left(p_{3}\right)$ these maps are distinct (a contradiction with the Riemann mapping theorem) unless $p_{2}=p_{3}$. But these points are determined by the conformal structure of the torus, so $\tau_{1}=\tau_{2}$. This shows that the ratio $\left|l_{2}\right| /\left|l_{1}\right|$ is monotone, and it is easy to check that it is increasing in $\operatorname{Im} \tau$.

Lemma 4.5. There exists a continuous curve $c: \mathbb{R} \rightarrow \mathbb{C}$ such that $\tau_{G} \in c(\mathbb{R})$ and $c(\mathbb{R}) \subset b^{-1}(0)$.

Proof. Since $b$ is continuous, $b<0$ on $Y$ and $b>0$ on $B$, the zero set of $b$ must topologically separate $B$ and $Y$; in particular, it contains a curve $c$ such that $\tau_{G} \in c$. This curve does not intersect $L$ or $R$, because there are no zeros on either. Thus $\operatorname{Im}(c(t)) \rightarrow 0$ or $\operatorname{Im}(c(t)) \rightarrow \infty$ as $t \rightarrow \pm \infty$.

Finally, the following proposition proves embeddedness.

Proposition 4.6. Let $M_{t}$ for $0 \leq t \leq 1$ be a continuously differentiable family of immersed triply periodic minimal surfaces. If $M_{0}$ is embedded, then $M_{t}$ is embedded for all $0 \leq t \leq 1$. 
Proof. Let $S$ be a fixed genus three Riemann surface. Then each $M_{t}$ can be parametrized (not conformally) by a map $f_{t}: S \rightarrow \mathbb{R}^{3}$. We write the family of surfaces as a map $f: S \times[0,1] \rightarrow \mathbb{R}^{3}$ by $f(x, t)=f_{t}(x)$ and assume that this map is continuously differentiable.

Let $t_{0}$ be the first time that a surface is not embedded, that is,

$$
t_{0}:=\inf \left\{t>0 \mid M_{t} \text { is not embedded }\right\} .
$$

We assume that $t_{0}$ exists and arrive at a contradiction.

We first prove that $M_{t_{0}}$ is not embedded. Let $t_{k} \rightarrow t_{0}$. Since $f\left(S, t_{k}\right)$ is not embedded, there exists $p_{k}, q_{k} \in S$ with $p_{k} \neq q_{k}$ such that $f\left(p_{k}, t_{k}\right)=f\left(q_{k}, t_{k}\right)$. Since $S$ is compact, there is a convergent subsequence of $p_{k}$ and $q_{k}$; without loss of generality we relabel to obtain sequences $p_{k} \rightarrow p$ and $q_{k} \rightarrow q$.

Case 1: $p=q$. Fix $\epsilon>0$. Since $f \in \mathscr{C}^{1}$ there exists $\delta_{1}>0$ and $N>0$ such that for all $k>N$ and for any $x \in B_{\delta_{1}}(p)$,

$$
\mid N\left(f\left(x, t_{k}\right)\right)-N\left(f\left(p, t_{0}\right) \mid<\epsilon .\right.
$$

Since $f(\cdot, t)$ is an immersion for each $t \in[0,1]$, we know $f\left(B_{r}(p), t_{k}\right)$ is an embedded minimal disk for sufficiently small $r$. Let

$$
\eta_{k}=\sup \left\{r>0 \mid f\left(B_{r}(p), t_{k}\right) \text { is an embedded minimal disk }\right\} .
$$

For sufficiently large $k$, we have $p_{k}, q_{k} \in B_{\delta}(p)$, and so $f\left(B_{\delta}(p), t_{k}\right)$ is not embedded for $k>N$ (possibly after increasing $N$ ). Thus $\eta_{k}<\delta$. Define $r_{k}=$ $\eta_{k}+\left(\delta-\eta_{k} / 2\right)$. Because $f\left(B_{r_{k}}, t_{k}\right)$ is not a graph over its tangent plane, there exist two points whose orthogonal projections to the tangent plane are the same. Therefore, by the mean value theorem, there must be some point $z_{k} \in B_{r_{k}}(p)$ such that $N\left(f\left(z_{k}, t_{k}\right)\right)$ is parallel to the tangent space at $p$, which contradicts (4-1), provided $\epsilon$ is sufficiently small.

Case 2: $p \neq q$. Since $f\left(p, t_{0}\right)=f\left(q, t_{0}\right)$ and $f\left(S, t_{0}\right)$ is embedded, for some $\delta>0$, we have $f\left(B_{\delta}(p), t_{0}\right)=f\left(B_{\delta}(q), t_{0}\right)$. This implies that $f\left(\cdot, t_{0}\right): S \rightarrow M_{t_{0}}$ is a mapping of degree 2; this is a contradiction since both $S$ and $M_{t_{0}}$ have genus three.

Having shown so far that $M_{t_{0}}$ is not embedded, we know it has a point of selfintersection. Also, $t_{0}>0$. Suppose that the planes tangent to the surface are transverse at the intersection point. Since transversality is an open condition: by the continuity of the family there exists an $\epsilon>0$ such that the tangent planes for $M_{t_{0}-\epsilon}$ will also be transverse. Therefore, $M_{t_{0}-\epsilon}$ has a self intersection, which contradicts the minimality of $t_{0}$. Thus the tangent planes must be coincident.

Suppose that $f\left(p_{1}, t_{0}\right)=f\left(p_{2}, t_{0}\right)$ is a point of self intersection. As above, $B_{r}\left(p_{i}\right)$ is an immersed minimal disk $M_{i}$ for sufficiently small $r>0$. Each is a minimal graph over their common tangent plane. Define a height function $h_{i}$ on 
$M_{i}$ as the height of $\operatorname{graph}\left(M_{i}\right)$. By the maximum principle for minimal surfaces, we cannot have $h_{1}-h_{2}>0$ on $B_{r}(p)-\{p\}$. Thus $h_{1}-h_{2}$ assumes some negative values. This being an open condition, there is an $\epsilon>0$ such that $h_{1}-h_{2}$ is also negative on a neighborhood of $G_{t_{0}-\epsilon}$. If $h_{1}-h_{2}$ is both negative and positive, the surface $G_{t_{0}-\epsilon}$ can not be embedded since the two graphs $M_{1}$ and $M_{2}$ intersect, which contradicts the minimality of $t_{0}$. Therefore, the family must be embedded for all $t>0$.

We can now prove the existence and embeddedness of the $t G$ family.

Proof of Theorem 1.2. By Lemma 4.5, there exists a family of tori such that the developed and normalized flat structures have the same vertical and horizontal turning angle $\theta$. We use the Gauss map used to develop these flat structures, and set $d h=e^{i \theta} d z$. This choice of height differential ensures that the flat structures are in the moduli spaces $\mathscr{V}(G)$ and $\mathscr{H}(G)$. Therefore, the period problem is solved for this Weierstrass data. The branched torus cover provides the conformal model of the triply periodic minimal surface, and the Gauss map $G$ and $d h$ that we have defined lift, via the rotation $\rho_{2}$, to a well-defined Gauss map and height differential for the triply periodic surface. This one-parameter family does contain the gyroid, by the description of the gyroid in Section 3.3. The entire family is embedded by Proposition 4.6.

\section{The $r G$ and $r L$ families}

5.1. Description of the Lidinoid. The $H$ surface (Figure 3.1) is a genus three triply periodic minimal surface that admits an order 3 rotational symmetry. It can be thought of as containing a "triangular catenoid" in the same way that the $P$ surface contains "square catenoids". Its lattice is spanned by a planar hexagonal lattice (along with a vertical component), in contrast to the square planar lattice for the $P$ surface. We proceed analogously to the $P$ surface and omit most details.

The order 3 rotation $\rho_{3}: \mathbb{R}^{3} \rightarrow \mathbb{R}^{3}$ descends on $H / \Lambda$ to a well-defined isometry with 2 fixed points. By Abel's theorem, there are only two possible locations for the pole of the Gauss map on the torus, $1 / 2$ or $1 / 2+\tau / 2$. Since there is a reflective symmetry of $H$ whose fixed point set contains both fixed points of the map, the same must be true on the torus. This forces the pole to be located at $1 / 2$. Again, symmetry considerations force the torus to be rectangular, and we normalize so that it is generated by $\langle 1, \tau\rangle$ for $\tau \in i \mathbb{R}$. The zero and poles are second-order by a computation similar to Lemma 2.9.

We write $G^{3}(z):=\rho \theta_{11}^{2}(z, \tau) / \theta_{11}^{2}(z-1 / 2, \tau)$ and we again set the Lopez-Ros factor $\rho=1$ for the appropriate normalizations. 


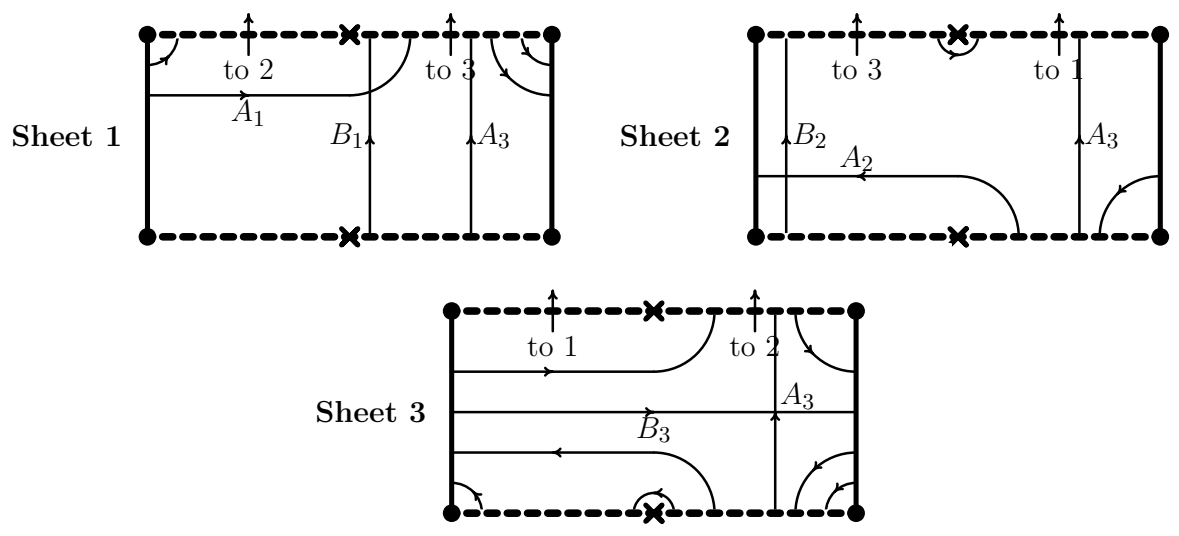

Figure 5.1. The conformal structure of the $H$ surface branched cover of a torus. Cuts are shown by dashed lines.

The torus is invariant under $-i d$, which is here the hyperelliptic involution. Figure 5.1 gives the conformal structure of the branched cover and the relevant cycles.

5.1.1. Flat structures. The 1 -forms $G d h,(1 / G) d h$, and $d h$ each place a flat structure on the torus which, after taking the quotient with $-i d$, descends to the sphere. We study each flat structure for the $H$ surface independently:

The $d h$ flat structure. Since this descends as $d z$, the flat structure for the torus is simply the rectangle.

The $G d h$ flat structure. Again, the order of the zeros and poles of the 1-form $G d h$ produce cone angles on the torus of $10 \pi / 3 i$ at 0 and of $2 \pi / 3$ at $1 / 2$. The remaining fixed points, at $\tau / 2$ and $1 / 2+\tau / 2$, are regular points. The involution $-i d$ halves the cone angles in the quotient, so that on the sphere have cone points of angle $10 \pi / 6$ at 0 , of angle $\pi / 3$ at $1 / 2$, and of angle $\pi$ at $\tau / 2$ and $1 / 2+\tau / 2$.

The flat structure is a hexagon:

Lemma 5.1. By cutting along the shortest geodesics on the sphere from $\tau / 2$ to 0 , 0 to $1 / 2$, and from $1 / 2$ to $1 / 2+\tau / 2$, we obtain a hexagon shown in black in Figure 5.2. The hexagon has the properties that

(i) the length of $l_{i}$ is equal to that of $l_{i}^{*}$ for $i=1,2,3$;

(ii) the angle between $l_{1}$ and $l_{2}$ and the angle between $l_{1}^{*}$ and $l_{2}^{*}$ are both $5 \pi / 6$;

(iii) the angle between $l_{1}$ and $l_{1}^{*}$ is $\pi / 2$ and the angle between $l_{3}$ and $l_{3}^{*}$ is $\pi$.

The proof is precisely analogous to Lemma 3.1. We find this flat structure inconvenient, given that the flat structure on the entire torus (without the $-i d$ identification) is so simple. The flat structure on the entire torus is obtained by 


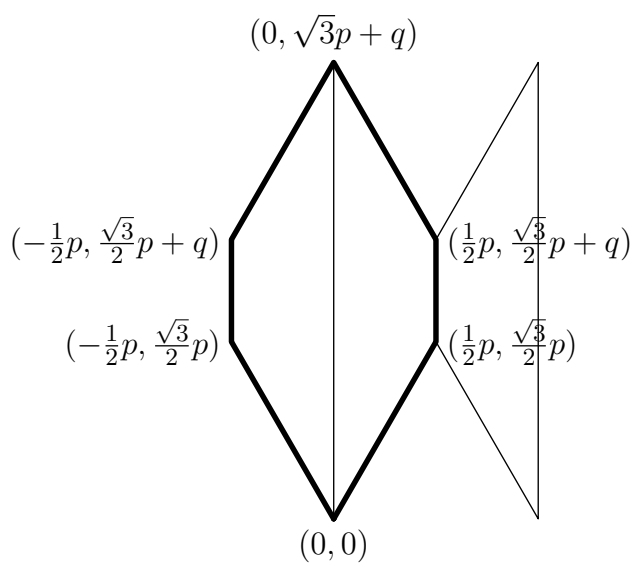

Figure 5.2. The $G d h$ (thick line) and $(1 / G) d h$ (thin line) flat structures for the $\mathrm{H}$ surface. Labeled vertices are for the $G d h$ flat structure (the corresponding points on $(1 / G) d h$ are obtained by translation by $(-1 / 2 p,-\sqrt{3} / 2 p)$.

rotating by $\pi$ about the vertex between $l_{3}$ and $l_{3}^{*}$ (the $-i d$ map descends on the developed image to the $-i d$ map since $1 / 2+\tau / 2$ is a regular point). Doing so, we obtain the flat structure shown in Figure 5.2; compare to the $P$ surface flat structure.

Again we have not chosen the imaginary part of $\tau$ as we expect to recover a family of surfaces.

We now describe the $(1 / G) d h$ flat structure for the $H$ surface. By precisely the same argument as in Section 3.1.3, it is simply a translate of the $G d h$ flat structure, with the Lopez-Ros factor $\rho=1$. The blue outline in Figure 5.2 shows this translation.

5.1.2. The period problem for the $H$ surface. We omit the computation of the periods, but we obtain

$$
\begin{array}{ll}
P\left(A_{1}\right)=(0,0,0), & P\left(B_{1}\right)=(0,-\sqrt{3} p-2 q, 0), \\
P\left(A_{2}\right)=(0,0,0), & P\left(B_{2}\right)=R_{2 \pi i / 3}(0,-\sqrt{3} p-2 q, 0), \\
P\left(A_{3}\right)=(0,0,0), & P\left(B_{3}\right)=(0,0,1) .
\end{array}
$$

Here the notation $R_{2 \pi i / 3}$ means a rotation by $2 \pi i / 3$ about the $x_{3}$-axis.

It is again immediate that these periods generate a 3-dimensional lattice $\Lambda$ for all values of $p$ and $q$. In other words, the period problem is solved no matter what the actual lengths of the segments in the developed flat structure are. Thus any value of a (and therefore, any quotient torus) solves the period problem. This proves that the $H$ surface comes in a 1-parameter family; in fact, the $H$ surface is in Meeks' 
family, so it comes in a 5-parameter family. This family is called the $\mathrm{rH}$ family. In the limit $\tau \rightarrow 0$, the $\mathrm{rH}$ surface looks like a pair of parallel planes joined with small catenoidal necks. As $\tau \rightarrow \infty$, it looks like three intersecting planes that are desingularized along the intersection by adding handles. (Compare this to the $t P$ family.)

To construct the Lidinoid, let $(X, G, d h)$ be the Weierstrass data describing a member of the $\mathrm{rH}$ family. As in the case of the gyroid, we can easily calculate the periods for all members of the associate family by using the $H$ surface flat structures. For instance, one can compute that for all $0 \leq \theta \leq 2 \pi$, the associate surface has periods

$$
P\left(A_{1}\right)=(\sqrt{3} p \sin \theta, p \sin \theta,--) \quad \text { and } \quad P\left(B_{3}\right)=(-\sqrt{3} p \sin \theta, p \sin \theta,--) .
$$

Since these two periods clearly generate the horizontal part of the lattice, we must ensure the others are compatible. For instance,

$$
P\left(B_{1}\right)=(\sqrt{3} p \sin \theta,-(2 q+\sqrt{3} p) \cos \theta,--) .
$$

Thus, since $\sin (\theta) \neq 0$ for nontrivial members of the associate family,

$$
\pm(2 q+\sqrt{3} p) \cos \theta=\sin \theta .
$$

Examining the periods for $B_{2}$ shows that we must choose the " + " equation, so that

$$
\theta=\arctan \left(\frac{-\sqrt{3} p-2 q}{p}\right) .
$$

Similarly to the gyroid, Equation (5-1) puts a constraint on $\theta$, and the vertical period condition places another condition; these two conditions are compatible for exactly one value of $\theta$ - the value that gives the Lidinoid.

The full set of periods of the Lidinoid for our parametrization are

$$
\begin{array}{ll}
P\left(A_{1}\right)=R_{2 \pi i / 3}(0,-1,0), & P\left(B_{1}\right)=-R_{4 \pi i / 3}(0,-1, s), \\
P\left(A_{2}\right)=-R_{2 \pi i / 3}(0,-1,0), & P\left(B_{2}\right)=-R_{4 \pi i / 3}(0,-1, s), \\
P\left(A_{3}\right)=(0,0,3 s), & P\left(B_{3}\right)=R_{4 \pi i / 3}(0,-1, s),
\end{array}
$$

where $s \in \mathbb{R}^{+}$is calculated with an elliptic integral.

\subsection{Moduli spaces for the rL family.}

5.2.1. Vertical moduli space $\mathscr{V}(L)$. The vertical moduli space is defined in precisely the same way as for the gyroid, that is, $\mathscr{V}(L)=\mathscr{V}(G)$. 

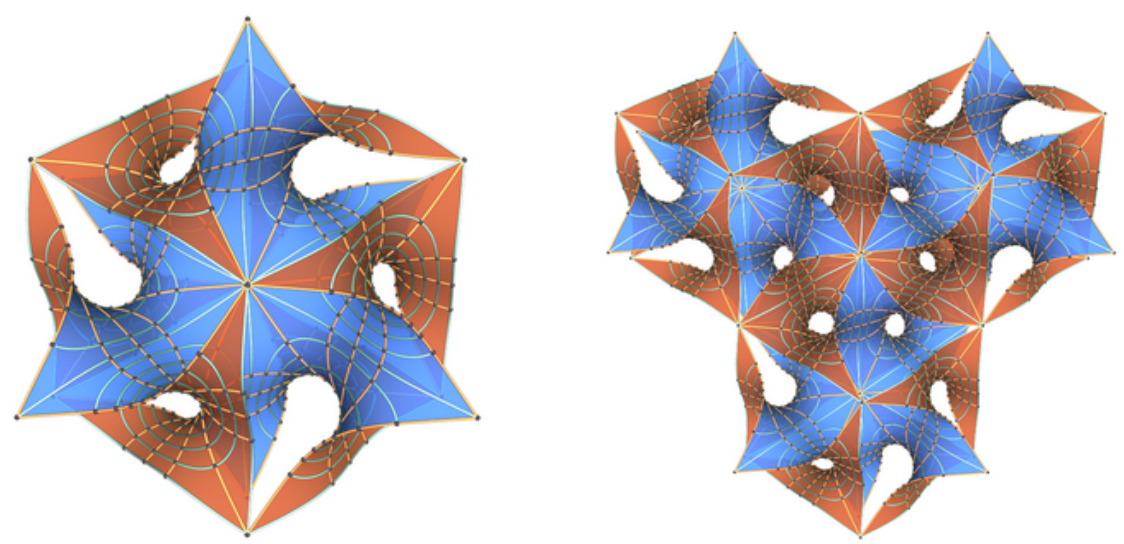

Figure 5.3. The Lidinoid.

5.2.2. Horizontal moduli space $\mathscr{H}(L)$. Suppose that $M$ is any immersed, genus three, triply periodic minimal surface that has as a conformal model a three-fold branched cover of a generic torus $\mathbb{C} / \Gamma$. Without loss of generality we write $\Gamma=\langle 1, \tau\rangle$. Suppose further that the square of the Gauss map descends to $\mathbb{C} / \Gamma$ and has a second-order pole at $1 / 2$ and a second-order zero at 0 . (This is the case for the Lidinoid, except that the torus is rectangular.) The quotient $S=\mathbb{C} / \Gamma /-i d$ is a sphere, and $G d h$ again induces a cone metric on $S$. Under this cone metric, the sphere is a tetrahedron, with vertex angle of $5 \pi / 3$ corresponding to the zero, a vertex angle of $\pi / 3$ corresponding to the pole, and two vertex angles of $\pi$ corresponding to the remaining fixed points $\tau / 2$ and $1 / 2+\tau / 2$ of $-i d$. The developed image of this sphere is parametrized as follows.

Lemma 5.2. For any torus $\mathbb{C} / \Gamma$ with $\Gamma=\langle 1, \tau\rangle$, the cone metric $G d h$ descends to $S$. By cutting along shortest geodesics on $S$ from $1 / 2$ to 0,0 to $\tau / 2$, and from $\frac{\tau}{2}$ to $\frac{1}{2}+\frac{\tau}{2}$, we obtain a hexagon. The hexagon has the properties that

(i) the length of $l_{i}$ is equal to that of $l_{i}^{*}$ for $i=1,2,3$;

(ii) $l_{2}$ is parallel to $l_{2}^{*}$;

(iii) the angle between $l_{1}$ and $l_{1}^{*}$ and the angle between $l_{3}$ and $l_{3}^{*}$ are both $\pi / 2$.

Since the fixed point $1 / 2+\tau / 2$ is regular before the application of -id, we can extend this to a developing map on the whole torus by rotating by $\pi$ about the intersection of $l_{4}$ and $l_{4}^{*}$. Doing this, we obtain the hexagon flat structure shown in Figure 5.4. We can parametrize this final space of possible hexagons by $\xi_{1}, \xi_{2} \in \mathbb{C}$ as shown in Figure 5.4.

Let $\widetilde{\mathscr{H}(G)}$ be the space of all hexagons satisfying the conditions of Lemma 5.2. The proof is precisely analogous to that of Lemma 4.1, and we omit the details. 


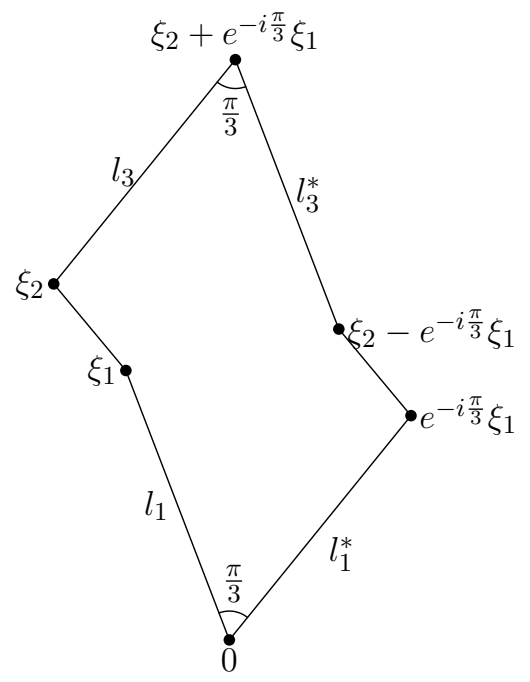

Figure 5.4. A generic member of the $\widetilde{\mathscr{H}(L)}$ moduli space.

To understand the horizontal periods, we again adjust $\rho$, if necessary, to normalize the $(1 / G) d h$ flat structure as in Section 3.1.3.

After the substitutions

$$
c=-\frac{1}{2}\left(\operatorname{Im} \xi_{1}+\sqrt{3} \operatorname{Re} \xi_{1}\right) \quad \text { and } \quad d=\frac{1}{2}\left(\operatorname{Im} \xi_{1}-4 \operatorname{Im} \xi_{2}+\sqrt{3} \operatorname{Re} \xi_{1}\right),
$$

the periods can be expressed as

$$
\begin{array}{ll}
P\left(A_{1}\right)=(\sqrt{3} c, c i, 0), & P\left(B_{1}\right)=(\sqrt{3} c, d, s), \\
P\left(A_{2}\right)=(-\sqrt{3} c,-c i, 0), & P\left(B_{2}\right)=(-(\sqrt{3} / 2)(d-c),-(1 / 2)(d-c), s), \\
P\left(A_{3}\right)=(0,0,3 s), & P\left(B_{3}\right)=(-\sqrt{3} c, c, s) .
\end{array}
$$

Again, $s$ is a factor determined by the torus. Since $P\left(B_{1}\right)=P\left(B_{2}\right)$ for the Lidinoid, we are forced to set $c=-d$ to solve the period problem. Therefore, the period problem is solved if $\operatorname{Im} \xi_{2}=0$. Note that, apart from parametrizing the flat structure differently (here 0 corresponds to a different cone angle on the $P$ surface), this is precisely the same condition as for the gyroid flat structures.

The same argument as before proves Theorem 1.3.

5.3. Description of the gyroid from the standpoint of the rPD family. In addition to the order 2 rotational symmetry, the standard, most symmetric $P$ surface also admits an order 3 symmetry. This symmetry permutes the handles of the $P$ surface and is obtained by rotating by $2 \pi / 3$ though the normal at one of the eight points where the Gaussian curvature $K=0$. We repeat the procedure discussed above for the $P$ surface (viewed as invariant under an order 2 rotation), and we obtain again 
a one-parameter family of $P$ surfaces, which this time is invariant under an order 3 rotation.

Since the standard $P$ surface is a member of the $r P D$ family, the gyroid can also be parametrized in terms of it. We outline the construction of the gyroid in this way, so that we can construct a second family of gyroids, the order 3 gyroids $r G$.

To begin, we need to find the conformal parameter $\tau$ that yields the standard, most symmetric $P$ surface. From the end of Section 3.3, recall that the standard $P$ surface can be described in terms of the 1-forms $\omega_{1}, \omega_{2}$, and $\omega_{3}$; these forms are considered with the orientation of the $P$ surface in space so that the lattice is the standard, cubical lattice. These are permuted by the rotation $\rho_{3}$. After a rotation of the surface in space so that the axis of rotation is vertical, $d h=\omega_{1}+\omega_{2}+\omega_{3}$. We understand the periods of these 1-forms explicitly from our work with the $P$ surface. Denote by $\gamma_{1}$ the cycle generated by the vector 1 on the order $2 P$ surface torus. Its period on each of the $\omega_{i}$ flat structures is 1 , that is, $\int_{\gamma_{1}} \omega_{i}=1$ so that $\int_{\gamma_{1}} d h=\int_{\gamma_{1}} \omega_{1}+\omega_{2}+\omega_{3}=1$. This implies that one generator of the quotient torus $P / \rho_{3}$ is 1 , since the location of the branch cuts implies that this cycle $\gamma_{1}$ continues onto all three sheets.

Considering the other generator of the torus (rather, the cycle $\gamma_{2}$ coinciding with this generator), we note that $\int_{\gamma_{2}} \omega_{i}=2 a$, since the cycle continues onto both sheets of the torus $P / \rho_{2}$ (recall that $a=\operatorname{Im} \tau \approx 0.78$ ). On the other hand, if we denote the generators of the torus $P / \rho_{3}$ by 1 and $\sigma$, then $\int_{\gamma_{2}} d h=\int_{\gamma_{2}} \omega_{1}+\omega_{2}+\omega_{3}=6 a$ but also $\int_{\gamma_{2}} d h=3 \operatorname{Im} \sigma$ since the cycles continues onto both sheets. Thus, the standard $P$ surface is obtained when $\sigma=2 \tau$. Since this is the standard $P$ surface, the angle of association that yields the gyroid is the same $\theta=\operatorname{arccot} \operatorname{Im} \tau$. This is precisely the same surface as obtained in Section 3.3, but viewed from a different perspective and using a different parametrization. Another view of the gyroid is in Figure 5.5.

\subsection{Moduli spaces for the rG family.}

5.4.1. Vertical moduli space $\mathscr{V}(r G)$. To obtain the standard gyroid from the order 3 perspective, we take as the torus parameter $\tau=2 \cdot a i$, where $a$ is the conformal parameter for the standard $P$ surface. We then use the same angle of association as in the order 2 parametrization, obtaining, $\operatorname{Re} e^{i \theta}=-2 \operatorname{Re} e^{i \theta} \tau$. We therefore define $\mathscr{V}(r G)=\left\{\left(\omega_{1}, \omega_{2}\right) \in \mathbb{C} \times \mathbb{C}|| \omega_{1} \mid=1\right.$ and $\left.\operatorname{Re} \omega_{1}=-2 \operatorname{Re} \omega_{2}\right\}$, so that the vertical period problem is solved.

5.4.2. Horizontal moduli space $\mathscr{H}(r G)$. Suppose $M$ is an immersed, genus three, triply periodic minimal surface that has as a conformal model a three-fold branched cover of a generic torus $\mathbb{C} / \Gamma$. Without loss of generality we write $\Gamma=\langle 1, \tau\rangle$. Suppose further that the square of the Gauss map descends to $\mathbb{C} / \Gamma$ and has a second-order pole at $1 / 2+\tau / 2$ and a second-order zero at 0 . (This is the case 

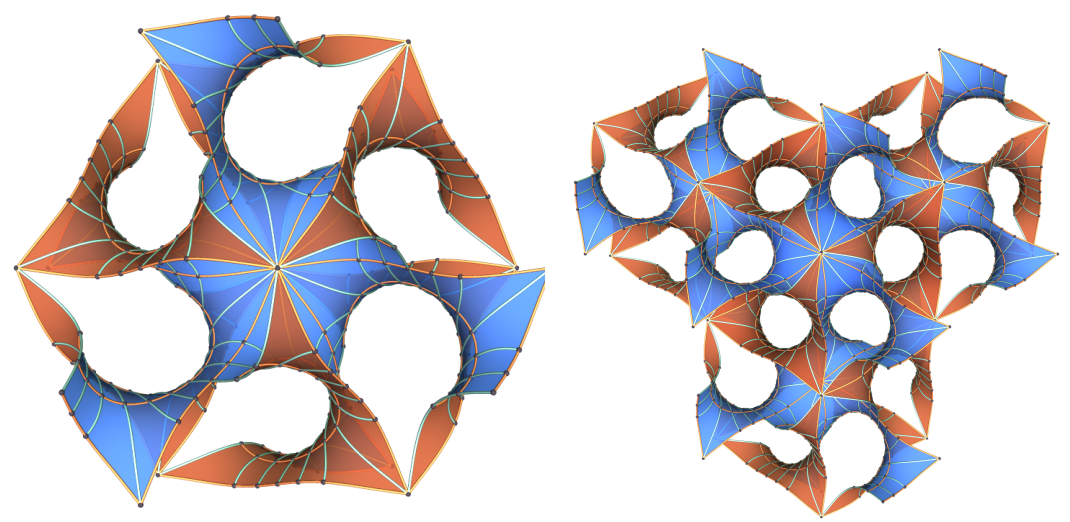

Figure 5.5. Left: a translational fundamental domain of the gyroid, viewed as invariant under an order 3 rotation. Right: several copies of a fundamental domain. Notice the similarity to the Lidinoid.

for the order 3 gyroid, except that the torus is rectangular.) The quotient $S=$ $\mathbb{C} / \Gamma /-i d$ is a sphere, and $G d h$ again induces a cone metric on $S$. Under this cone metric, the sphere is a tetrahedron, with vertex angle of $5 \pi / 3$ corresponding to the zero, a vertex angle of $\pi / 3$ corresponding to the pole, and two vertex angles of $\pi$ corresponding to the remaining fixed points $\tau / 2$ and $1 / 2$ of $-i d$. The developed image of this sphere is described as follows.

Lemma 5.3. For any torus $\mathbb{C} / \Gamma$ with $\Gamma=\langle 1, \tau\rangle$, the cone metric $G d h$ descends to $S$. By cutting along shortest geodesics on $S$ from $1 / 2$ to 0 , from 0 to $\tau / 2$, and from $\tau / 2$ to $1 / 2+\tau / 2$, we obtain a hexagon shown in Figure 5.6. The hexagon has properties that

(i) the length of $l_{i}$ is equal to that of $l_{i}^{*}$ for $i=1,2,3$;

(ii) $l_{2}=e^{i 2 \pi / 3} l_{2}^{*}$;

(iii) the angle between $l_{1}$ and $l_{1}^{*}$ is $\pi$ and the angle between $l_{3}$ and $l_{3}^{*}$ is $\pi / 3$.

We can parametrize this final space of possible hexagons by $\xi_{1}, \xi_{2} \in \mathbb{C}$ as shown in Figure 5.6. We use the notation $\widehat{\xi_{1}}=e^{-i \pi / 3}\left(\xi_{2}-\xi_{1}\right)+\xi_{1}-e^{-2 \pi i / 3} \xi_{1}$.

We call the space of all hexagons satisfying the conditions of Lemma $5.3 \widetilde{\mathscr{H}(r G)}$.

To understand the horizontal periods, we again adjust $\rho$, if necessary, to normalize the $(1 / G) d h$ flat structure as in Section 3.1.3. Then in terms of these flat 


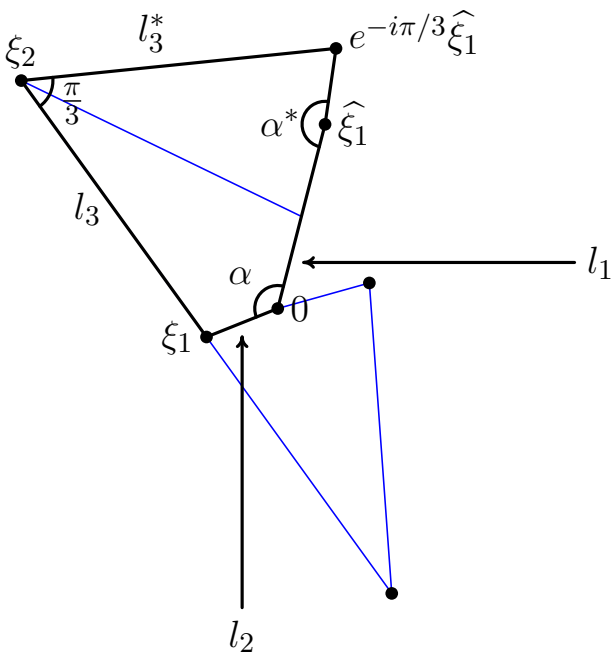

Figure 5.6. A generic member of the $\mathscr{H}(r G)$ moduli space.

structures, we compute the periods of the six generators of $H_{1}(M, Z)$ to be

$$
\begin{array}{ll}
P\left(A_{1}\right)=(a, 0,-s), & P\left(B_{1}\right)=(b, \sqrt{3}(a-b), 2 s), \\
P\left(A_{2}\right)=(a, 0,-s), & P\left(B_{2}\right)=(b, \sqrt{3}(a-b), 2 s), \\
P\left(A_{3}\right)=(a, 0,-s), & P\left(B_{3}\right)=(b, \sqrt{3}(a-b), 2 s) .
\end{array}
$$

Here we simplified the expressions using the substitutions

$$
\begin{aligned}
& a=2 \sqrt{3} \operatorname{Im} \xi_{1}-\sqrt{3} \operatorname{Im} \xi_{2}+2 \operatorname{Re} \xi_{1}-3 \operatorname{Re} \xi_{2}, \\
& b=\sqrt{3} \operatorname{Im} \xi_{1}-(\sqrt{3} / 2) \operatorname{Im} \xi_{2}+\operatorname{Re} \xi_{1}-(5 / 2) \operatorname{Re} \xi_{2} .
\end{aligned}
$$

Again, $s$ is a factor determined by the torus. Since, for the gyroid, one computes that $P\left(A_{1}\right)=P\left(B_{2}\right)$, we must set $a=b$ to solve the period problem. Therefore, the period problem is solved if

$$
\sqrt{3} \operatorname{Im} \xi_{1}-(\sqrt{3} / 2) \operatorname{Im} \xi_{2}+\operatorname{Re} \xi_{1}-(1 / 2) \operatorname{Re} \xi_{2}=0 .
$$

This seemingly complicated expression is actually very reasonable; it holds if and only if $\arg \left(\widehat{\xi}_{1}-\xi_{2}\right)=\pi / 3$. But the consequence of this is that any member of $\widehat{\mathscr{H}(r G)}$ solves the period problem after a rotation (and so we can again define the relative turning angles). We define

$$
\mathscr{H}(r G)=\left\{\left(\xi_{1}, \xi_{2}\right) \in \widehat{\mathscr{H}(r G)} \mid \sqrt{3} \operatorname{Im} \xi_{1}-(\sqrt{3} / 2) \operatorname{Im} \xi_{2}+\operatorname{Re} \xi_{1}-(1 / 2) \operatorname{Re} \xi_{2}=0\right\} .
$$

The rest of the proof of the existence of a family of order 3 gyroids is analogous to the other two families discussed above, and this proves Theorem 1.4. 


\title{
References
}

[Bonnet 1853] O. Bonnet, "Deuxième note sur les surfaces à lignes de courbure spheŕiques.", C.R. Acad. Sci. Paris 36 (1853), 389-91, 585-7.

[Dierkes et al. 1992] U. Dierkes, S. Hildebrandt, A. Küster, and O. Wohlrab, Minimal surfaces. I. Boundary value problems, Grundlehren der Mathematischen Wissenschaften 295, Springer, Berlin, 1992. MR 94c:49001a Zbl 0777.53012

[Farkas and Kra 1992] H. M. Farkas and I. Kra, Riemann surfaces, Second ed., Graduate Texts in Mathematics 71, Springer, New York, 1992. MR 93a:30047 Zbl 0764.30001

[Fogden and Hyde 1999] A. Fogden and S. T. Hyde, "Continuous transformations of cubic minimal surfaces", Eur. Phys. J. B 7:1 (1999), 91-104.

[Fogden et al. 1993] A. Fogden, M. Haeberlein, and S. Lidin, "Generalizations of the gyroid surface”, J. Physique I 3:12 (1993), 2371-2385. MR 94h:53010

[Große-Brauckmann and Wohlgemuth 1996] K. Große-Brauckmann and M. Wohlgemuth, "The gyroid is embedded and has constant mean curvature companions", Calc. Var. Partial Differential Equations 4:6 (1996), 499-523. MR 97k:53011 Zbl 0930.53009

[Karcher 1989] H. Karcher, "The triply periodic minimal surfaces of Alan Schoen and their constant mean curvature companions”, Manuscripta Math. 64:3 (1989), 291-357. MR 90g:53010 Zbl 0687.53010

[Lidin and Larsson 1990] S. Lidin and S. Larsson, "Bonnet transformation of infinite periodic minimal surfaces with hexagonal symmetry", J. Chem. Soc. Faraday Trans. 86:5 (1990), 769-775.

[López and Martín 1999] F. J. López and F. Martín, "Complete minimal surfaces in $\mathbf{R}^{3}$ ", Publ. Mat. 43:2 (1999), 341-449. MR 2002c:53010 Zbl 0951.53001

[López and Ros 1991] F. J. López and A. Ros, "On embedded complete minimal surfaces of genus zero", J. Differential Geom. 33:1 (1991), 293-300. MR 91k:53019 Zbl 0719.53004

[Meeks 1975] W. H. Meeks, III, The Geometry and the Conformal Structure of Triply Periodic Minimal Surfaces in $\mathbb{R}^{3}$, PhD thesis, University of California, Berkeley, 1975.

[Nitsche 1975] J. C. C. Nitsche, Vorlesungen über Minimalfächen, Grundlehren der mathematischen Wissenschaften 199, Springer, Berlin, 1975. MR 56 \#6533 Zbl 0319.53003

[Osserman 1969] R. Osserman, A survey of minimal surfaces, Van Nostrand Reinhold Co., New York, 1969. MR 41 \#934 Zbl 0209.52901

[Schoen 1970] A. H. Schoen, "Infinite periodic minimal surfaces without self-intersections", Technical note TN D-5541, NASA, 1970.

[Traizet 2008] M. Traizet, "On the genus of triply periodic minimal surfaces", J. Diff. Geom. (2008).

To appear.

[Weber 1998] M. Weber, "On the Horgan minimal non-surface", Calc. Var. Partial Differential Equations 7:4 (1998), 373-379. MR 99m:53019 Zbl 1008.53009

Received May 31, 2007. Revised July 6, 2007.

\author{
ADAM G. WEYHAUPT \\ DePARTMENT OF MATHEMATICS AND STATistics, Box 1653 \\ SOUTHERN ILLINOIS UNIVERSITY \\ EDWARDSVILLE, IL 62026 \\ UNITED STATES \\ aweyhau@siue.edu \\ http://www.siue.edu/ aweyhau
}

